TRANSACTIONS OF THE

AMERICAN MATHEMATICAL SOCIETY

Volume 362, Number 10, October 2010, Pages 5189-5211

S 0002-9947(2010)04890-1

Article electronically published on May 20, 2010

\title{
GENERIC HYPERBOLICITY OF EQUILIBRIA AND PERIODIC ORBITS OF THE PARABOLIC EQUATION ON THE CIRCLE
}

\author{
ROMAIN JOLY AND GENEVIÈVE RAUGEL
}

\begin{abstract}
In this paper, we show that, for scalar reaction-diffusion equations on the circle $S^{1}$, the property of hyperbolicity of all equilibria and periodic orbits is generic with respect to the non-linearity. In other words, we prove that in an appropriate functional space of non-linear terms in the equation, the set of functions, for which all equilibria and periodic orbits are hyperbolic, is a countable intersection of open dense sets. The main tools in the proof are the property of the lap number and the Sard-Smale Theorem.
\end{abstract}

\section{INTRODUCTION}

In the local study of the qualitative properties of perturbed dynamical systems, the concepts of non-degeneracy and hyperbolicity of equilibria and periodic orbits play a crucial role. Indeed, if one slightly perturbs a continuous dynamical system whose equilibria and periodic orbits are non-degenerate, then at least the perturbed system still admits equilibria and periodic orbits nearby. If these elements are in addition hyperbolic, then no local bifurcation phenomena occur, which means that the dynamics in the neighborhood of hyperbolic equilibria and periodic orbits is stable under small perturbations. This stability property has practical consequences. For example, it implies that a numerical simulation of the dynamics near a hyperbolic equilibrium or periodic orbit is qualitatively correct. These considerations show that it is important to know if, in a given class of dynamical systems or evolutionary equations, the equilibria and periodic orbits are all hyperbolic or if, at least, this property is generically satisfied.

Generic hyperbolicity of equilibrium points and periodic orbits is well known in the case of diffeomorphisms or vector fields on a compact finite-dimensional manifold (for instance, see Chapter 3 of [24] and the references therein). The genericity of hyperbolicity of equilibria and periodic orbits was generalized in 1977 to the case of functional differential equations defined in $\mathbb{R}^{n}, n \geq 1$, by Mallet-Paret ([21]). In the frame of evolutionary partial differential equations, generic hyperbolicity of equilibrium points is also a classical result (see for example, [4, 6], 7] or [30 for parabolic equations). Notice that this is sufficient for gradient dynamical systems, where no periodic orbits can occur. On the contrary, for autonomous evolutionary

Received by the editors May 20, 2008.

2010 Mathematics Subject Classification. Primary 35B10, 35B30, 35K57, 37D05, 37D15, 37L45; Secondary 35B40.

Key words and phrases. Hyperbolicity, genericity, periodic orbits, equilibria, Sard-Smale, lap number.

(C)2010 American Mathematical Society Reverts to public domain 28 years from publication 
partial differential equations, which are not of gradient type, the hyperbolicity of periodic orbits seems to be an open question.

In this paper, we address the question of genericity of hyperbolicity of periodic orbits by considering one of the simplest non-gradient partial differential equations, namely the following scalar reaction-diffusion equation on the one-dimensional torus (or unit circle) $S^{1}=\mathbb{R} / 2 \pi \mathbb{Z}$, given by

$$
\begin{aligned}
u_{t}(x, t) & =u_{x x}(x, t)+f\left(x, u(x, t), u_{x}(x, t)\right), \quad(x, t) \in S^{1} \times \mathbb{R}_{+}^{*}, \\
u(x, 0) & =u_{0}(x), \quad x \in S^{1},
\end{aligned}
$$

where $f$ belongs to the space $C^{2}\left(S^{1} \times \mathbb{R} \times \mathbb{R}, \mathbb{R}\right)$ and $u_{0}$ is given in the Sobolev space $H^{s}\left(S^{1}\right)$, with $s \in(3 / 2,2)$ (so that $H^{s}\left(S^{1}\right)$ is continuously embedded into $C^{1+\alpha}\left(S^{1}\right)$ for $\left.\alpha=s-3 / 2\right)$. Our purpose is to prove that the equilibria and the periodic orbits of (1.1) are hyperbolic generically with respect to $f$. We will also consider the more constricting case where the non-linearity is independent of $x$ :

$$
u_{t}(x, t)=u_{x x}(x, t)+f\left(u(x, t), u_{x}(x, t)\right), \quad(x, t) \in S^{1} \times \mathbb{R}_{+}^{*} .
$$

Equations (1.1) and (1.2) are good problems to begin with. Indeed, on one hand these equations may have periodic orbits (see Theorem 2 of 29 ), and on the other hand they are the simplest non-gradient PDE's to study, since they admit very special properties as explained below.

In the study of global stability, generic hyperbolicity of closed orbits also plays an important role. Showing genericity of closed orbits is the first step in the proof of the genericity of Morse-Smale property. Morse-Smale dynamical systems are systems whose non-wandering set consists only in a finite number of hyperbolic equilibria and hyperbolic periodic orbits and for which the intersections of the stable and unstable manifolds of equilibria and periodic orbits are all transversal. On finitedimensional compact manifolds, the dynamics of Morse-Smale dynamical systems are globally stable under perturbations; that is, any small regular enough perturbation of a Morse-Smale system is still Morse-Smale, and the flows are topologically equivalent (for more details, see [24). In the class of gradient dynamical systems on finite-dimensional compact manifolds, the Morse-Smale property is known to be generic. For the non-gradient dynamical systems, the Morse-Smale property is generic only if the manifold is one- or two-dimensional. In the infinite-dimensional case, the structural stability of Morse-Smale systems remains true under compactness assumptions (for instance, under the assumption that the dynamical systems admit compact global attractors). The genericity of Morse-Smale property has been obtained for some classes of gradient partial differential equations ([1, 16, 7, 8, 18). For Morse-Smale non-gradient evolutionary partial differential equations, Equation (1.1) is the right candidate, since its asymptotic behaviour in time is analogous to the one of a system of ordinary differential equations in $\mathbb{R}^{2}$. Indeed, in [10, Fiedler and Mallet-Paret have proved that Equation (1.1) satisfies a Poincaré-Bendixson type property. For non-linearities independent of $x$, automatic transversality properties have been proved in [13, by showing that, for (1.2), the Morse-Smale property is equivalent to the hyperbolicity of all closed orbits. The results of the present paper thus imply the genericity of Morse-Smale property for Equation (1.2). In the more general case of Equation (1.1), R. Czaja and C. Rocha have shown in 9] that, if the periodic orbits of (1.1) are all hyperbolic, then their stable and unstable manifolds intersect transversally. Here we will show that this hyperbolicity is generic in 
the non-linearity $f\left(x, u, u_{x}\right)$. This shows, together with the results of [9], that the transversality of the stable and unstable manifolds of the periodic orbits of (1.1) is generic with respect to $f$. Thus, this paper is also a further step towards the proof of genericity of the Morse-Smale property for (1.1). We shall conclude this proof in the forthcoming paper [20].

When the non-linearity $f$ does not explicitly depend on $x$ and is dissipative, the dynamics of Equation (1.2) can be described with more precision. In [3], Angenent and Fiedler obtained the following characterization of the closed orbits of (1.2).

Proposition 1.1. Any closed orbit $u(x, t)$ of (1.2) is: 0 ,

- either a homogeneous equilibrium point; that is, $u(x, t) \equiv e \in \mathbb{R}$, where $f(e, 0)=$

- or a rotating wave; that is, $u(x, t)=v(x-c t)$ with $c \neq 0$,

- or a frozen wave; that is, $u$ is a non-homogeneous solution of $u_{x x}+f\left(u, u_{x}\right)=0$.

Moreover, Angenent and Fiedler have proved that the $\omega$-limit set of any element $u_{0} \in H^{s}\left(S^{1}\right)$ contains a rotating wave or a steady state. Under the hypothesis that the rotating waves and equilibria are all hyperbolic, they showed that the $\omega$-limit set consists exactly in one rotating wave or in one equilibrium point and they also described the connecting orbits. In [23], Matano and Nakamura proved that there exist neither homoclinic orbits nor heteroclinic cycles. In [13], Fiedler, Rocha and Wolfrum, using the notion of "adjacency", have characterized connecting orbits under the hypothesis that the rotating waves and equilibria are hyperbolic. They also gave an equivalent characterization of the property of hyperbolicity of rotating waves. Thus, the generic hyperbolicity of the closed orbits of (1.2) is primordial to conclude that the dynamics described in these papers include all possible dynamics, except maybe some exceptional ones.

Finally, let us recall that, if the periodic boundary conditions are replaced by homogeneous Dirichlet or Neumann boundary ones, then the dynamical system $S(t)$ associated with (1.1) is of gradient type and the $\omega$-limit set of any point is exactly one equilibrium point (see [32]). Moreover, in these cases, as shown by D. Henry [16] and later by S. Angenent [1, the stable and unstable manifolds of the equilibria intersect transversally, provided they are all hyperbolic. Thus, the Morse-Smale property is generic in the class of one-dimensional scalar parabolic equations on the segment with Dirichlet or Neumann boundary conditions. In a series of papers, Fiedler and Rocha have described the different equivalence classes of attractors and, in particular, the heteroclinic orbits connecting the equilibria (see [11, [12, for example).

Now we are going to precisely state the genericity results proved in this paper. We set $\mathfrak{G}=\mathcal{C}^{2}\left(S^{1} \times \mathbb{R} \times \mathbb{R}, \mathbb{R}\right)$ and we endow $\mathfrak{G}$ with the Whitney topology; that is, the topology generated by the neighborhoods

$\left\{g \in \mathfrak{G} /\left|D^{i} f(x, u, v)-D^{i} g(x, u, v)\right| \leq \delta(u, v), \forall i \in\{0,1,2\}, \forall(x, u, v) \in S^{1} \times \mathbb{R}^{2}\right\}$,

where $f$ is any function in $\mathfrak{G}$ and $\delta$ is any positive continuous function (see [14]).

It is well known that $\mathfrak{G}$ is a Baire space, which means that any generic set, that is, any countable intersection of open and dense sets, is dense in $\mathfrak{G}$ (see [14] for instance). 
In the general case, when $f$ also depends on the variable $x$, we prove the following genericity result (for the definition of hyperbolicity we refer the reader to Section 2 below).

Theorem 1.2. There exists a generic subset $\mathcal{O}$ of $\mathfrak{G}$ such that, for all $f \in \mathcal{O}$, all the equilibrium points and all the periodic solutions of (1.1) are hyperbolic.

Remarks. 1) The above theorem is also valid if, in the equation (1.1), we replace the Laplacian $-\partial_{x x}$ by the self-adjoint operator $-a(x) \partial_{x x}$ or $-a(x)^{-1}\left(\partial_{x}\left(a(x) \partial_{x} \cdot\right)\right)$, where $a(x)>0$ is a $C^{1}$-function on $S^{1}$.

2) In Theorems 1.2 and 1.3, we assume for sake of simplicity that $f$ is of class $C^{2}$ in all the variables. Actually, we can begin with a non-linearity $f$, which is less regular, and immediately replace it by a small perturbation $f_{0}$, which is in $C^{2}\left(S^{1} \times \mathbb{R} \times \mathbb{R}, \mathbb{R}\right)$.

As we already mentioned, it is also interesting to consider a non-linearity $f$, which is independent of $x$. Even if the choice of such non-linearity is more constrained, this case is not more difficult than the general case due to the particular properties coming from the $S^{1}$-equivariance of (1.2). In Section 5, we prove the following genericity result.

Theorem 1.3. Let $\mathfrak{G}^{i}$ be the subspace of $\mathfrak{G}$ consisting of functions independent of $x$. There exists a generic subset $\mathcal{O}^{i}$ of $\mathfrak{G}^{i}$ such that, for all $f \in \mathcal{O}^{i}$, (1.2) has no frozen waves and any homogeneous equilibrium or rotating wave of (1.2) is hyperbolic.

Remark. In [13, the generic hyperbolicity of homogeneous equilibria and rotating waves is asserted, based on a personal communication of P. Brunovský.

There are two main ingredients in the proof of Theorems 1.2 and 1.3 . The first one, as expected in genericity properties, is the Sard-Smale Theorem that we recall in the appendix. The second main ingredient is the Sturm property (also called a zero number or lap number property). This property originated in the paper of Sturm [31] (see also 22] and 22 for more recent results, mainly in the case where $f$ is not analytic). For any $\varphi \in C^{1}\left(S^{1}\right)$, we define the zero number $z(\varphi)$ as the (even) number of strict sign changes of $\varphi$. Let $I$ be an open subinterval of $\mathbb{R}$ and let $v(x, t)$ be a solution of the linear parabolic equation

$$
v_{t}=v_{x x}+b(x, t) v+d(x, t) v_{x},
$$

where $b, b_{x}, b_{t}$ and $d$ are bounded functions on any compact subset of $S^{1} \times I$. Then $z(v(\cdot, t))$ is finite, for any $t>0$, and non-increasing with $t$. Moreover, the zero number $z(v(\cdot, t))$ drops strictly at $t=t_{0}$, if and only if there exists $x_{0} \in S^{1}$ such that

$$
v\left(x_{0}, t_{0}\right)=0, \quad \partial_{x} v\left(x_{0}, t_{0}\right)=0 .
$$

In Section 2.3, we will see that these remarkable properties of the zero number also imply special spectral properties for the linearized equation associated with (1.1). In the proof of Theorem 1.2, it will also play an important role.

Finally, we point out that the zero number property has been widely used in all the above mentioned papers dealing with the description of the dynamics of a scalar one-dimensional equation. 
The paper is organized as follows. In Section 2, we give the main spectral properties of the linearized equations around the equilibria and the periodic solutions of (1.1). Section 3 contains the proof of the genericity of the hyperbolicity of the equilibria of (1.1), while Section 4 is devoted to the genericity of the hyperbolicity of the periodic orbits of (1.1). In Section 5, we consider the special case of equation (1.2) and prove Theorem 1.3. Finally, in the appendix, we recall the Sard-Smale Theorem, that we apply here.

\section{Spectrum of the Linearized operators}

The notions of non-degeneracy and hyperbolicity of equilibria and periodic orbits of an evolutionary equation are related to the spectral properties of the linearized equations around these elements. In this section, we will recall these notions and describe several properties of the linearized equations associated with (1.1).

Let $p$ be either an equilibrium point $e$ or a periodic orbit $p_{0}(x, t)$ of (1.1). We consider the linearized equation

$$
\begin{aligned}
\varphi_{t} & =\varphi_{x x}+D_{u} f\left(x, p, p_{x}\right) \varphi+D_{u_{x}} f\left(x, p, p_{x}\right) \varphi_{x}, \\
\varphi(x, 0) & =\varphi_{0}(x) .
\end{aligned}
$$

Let $s \in(3 / 2,2)$; we introduce the operator $U(t, 0): H^{s}\left(S^{1}\right) \longrightarrow H^{s}\left(S^{1}\right)$, defined by $U(t, 0) \varphi_{0}=\varphi(t)$, where $\varphi(t)$ is the solution of the linearized equation (2.1).

Sometimes, if needed, we will also denote the operator $U(t, 0)$ by $U_{p, f}(t, 0)$.

2.1. The case of equilibria. Let $e \in H^{s}\left(S^{1}\right)$ be an equilibrium point of (1.1), that is, a solution of $e_{x x}+f\left(x, e, e_{x}\right)=0$. Due to the classical elliptic estimates and the regularity of $f$, the equilibrium $e$ belongs to $H^{s+2}\left(S^{1}\right)$. In the case where $p=e$ is an equilibrium point, the linearized operator $U(t, 0)$ is an analytic semigroup generated by the linear operator

$$
L_{e}=\partial_{x x}^{2}+f_{u}^{\prime}\left(x, e, e_{x}\right)+f_{u_{x}}^{\prime}\left(x, e, e_{x}\right) \partial_{x}: H^{2}\left(S^{1}\right) \rightarrow L^{2}\left(S^{1}\right) ;
$$

that is, $U(t, 0)=e^{L_{e} t}$.

The general definitions of simplicity and hyperbolicity are as follows.

Definition 2.1. 1) We say that an equilibrium point $e$ is simple if 0 belongs to the resolvent set of the operator $L_{e}$.

2) We say that $e$ is a hyperbolic equilibrium point if the intersection of the spectrum $\sigma(U(t, 0))$ of $U(t, 0)$ with the unit circle $S^{1}$ in the complex plane is the empty set.

Since $L_{e}$ is a Fredholm operator and also a sectorial operator, simple characterizations of the notions of simplicity and hyperbolicity can be given (for the properties of Fredholm operators, see for example [5]).

Lemma 2.2. Let $e$ be any equilibrium point of (1.1).

1) The operator $L_{e}: H^{2}\left(S^{1}\right) \longrightarrow L^{2}\left(S^{1}\right)$ is a Fredholm operator of index 0 . As a consequence, we have the following obvious equivalences:

$$
\text { e is simple } \Leftrightarrow \operatorname{Ker} L_{e}=\{0\} \Leftrightarrow L_{e} \text { is surjective. }
$$

2) $U(t, 0): H^{s}\left(S^{1}\right) \longrightarrow H^{s}\left(S^{1}\right)$ is compact, and so $e$ is hyperbolic $\Leftrightarrow$ no eigenvalue of $L_{e}$ belongs to the imaginary axis. 
3) The operator $L_{e}$ is a sectorial operator with compact resolvent. Moreover, the sector is locally uniform in the sense that, for any $f \in \mathfrak{G}$ and any equilibrium point $e$ of (1.1), there exist positive constants $C$ and $C^{\prime}$ uniform in a neighborhood of $(e, f)$ in $H^{s}\left(S^{1}\right) \times \mathfrak{G}$ such that, for any eigenvalue $\lambda$ of $L_{e}$,

$$
|\operatorname{Im}(\lambda)| \leq C-C^{\prime} \operatorname{Re}(\lambda) .
$$

Proof. 1) Since $e \in H^{2}\left(S^{1}\right), \varphi \longmapsto \varphi+f_{u}^{\prime}\left(x, e, e_{x}\right) \varphi+f_{u_{x}}^{\prime}\left(x, e, e_{x}\right) \varphi_{x}$ is bounded from $H^{2}\left(S^{1}\right)$ into $H^{1}\left(S^{1}\right)$. Thus, $L_{e}$ is a compact perturbation of $\partial_{x x}^{2}-I d$ : $H^{2}\left(S^{1}\right) \rightarrow L^{2}\left(S^{1}\right)$. Since $\partial_{x x}^{2}-I d$ is bijective, it is a Fredholm operator of index 0 , which implies that $L_{e}$ is also a Fredholm operator of index 0 .

2) Since $L_{e}: H^{2}\left(S^{1}\right) \rightarrow L^{2}\left(S^{1}\right)$ is a real operator with compact resolvent, its spectrum consists in a sequence of eigenvalues of finite multiplicity and its spectrum is symmetric with respect to the real axis. Due to the smoothing properties of the parabolic flow, $e^{L_{e}}$ is a compact operator, thus its spectrum consists in $\{0\}$ and a sequence of eigenvalues with finite multiplicity converging to 0 . Hence, due to the spectral mapping theorem (see theorems 2.2.3 and 2.2.4 of [25] for example),

$$
\exp \left(t \sigma\left(L_{e}\right)\right) \subset \sigma\left(e^{L_{e} t}\right) \subset \exp \left(t \sigma\left(L_{e}\right)\right) \cup\{0\}, \quad \forall t \geq 0,
$$

which clearly implies the equivalence.

3) Moreover, $L_{e}$ is the perturbation of the self-adjoint operator $\partial_{x x}^{2}+f_{u}^{\prime}\left(x, e, e_{x}\right)$ by the term $f_{u_{x}}\left(x, e, e_{x}\right) \partial_{x}$. Therefore, it is a sectorial operator by Theorem 3.2.1 of [25] or by Theorem 1.3.2 of [15]. The fact that the sector is locally uniform in $(e, f)$ and the estimate (2.3) are straightforward consequences of the proof of Theorem 1.3.2 of [15].

In Section 2.3 below, we will prove special spectral properties of general scalar one-dimensional linear parabolic equations. As a direct consequence of the spectral theorem and of Theorem 2.6 and Proposition 2.7 below, we obtain the following interesting spectral property, which we will apply in the proof of the genericity theorems.

Proposition 2.3. Let $e$ be any equilibrium point of (1.1). Then, the dimension of the generalized eigenspace corresponding to the eigenvalues of $L_{e}$ with the same real part is at most two. Moreover, if $\lambda$ is a real eigenvalue of $L_{e}$, then there exists no other eigenvalue of $L_{e}$ with real part equal to $\lambda$.

2.2. The case of periodic orbits. In this section, we consider the non-trivial time-periodic solutions of Equation (1.1). Let $p=p_{0}(x, t)$ be a periodic solution of (1.1) of period $T_{0}>0$. We recall that $U(t, 0) \equiv U_{f}(t, 0)$ denotes the linear operator associated with (2.1) and that, since $p_{0}(t)$ is of period $T_{0}, U\left(T_{0}, 0\right)$ is called the period map.

Notice that, since $p_{0}(t)$ is a periodic solution with period $T_{0}$ of the autonomous equation (1.1), $\mu=1$ is automatically an eigenvalue of $U\left(T_{0}, 0\right)$ with eigenfunction $\partial_{t} p_{0}(0)$.

Definition 2.4. Let $p_{0}(t)$ be a periodic solution of (1.1), with period $T_{0}>0$.

1) We say that $p_{0}(t)$ is a simple or non-degenerate periodic orbit if the eigenvalue $\mu=1$ is simple and isolated from the rest of the spectrum. Then, $T_{0}$ is called a simple period. 
2) We say that $p_{0}(t)$ is hyperbolic if the eigenvalue $\mu=1$ is simple and if the intersection of the spectrum of $U\left(T_{0}, 0\right)$ with the unit circle reduces to the eigenvalue 1.

Remark that, since Equation (2.1) is smoothing in finite time, $U\left(T_{0}, 0\right)$ is a compact map from $H^{s}\left(S^{1}\right)$ into itself. Therefore, the spectrum of $U\left(T_{0}, 0\right)$ consists of 0 and a sequence of eigenvalues $\mu_{k}$ converging to 0 . Thus, $p_{0}(t)$ is a hyperbolic periodic orbit if $\mu=1$ is the only eigenvalue of $U\left(T_{0}, 0\right)$ on the unit circle and is simple.

Several properties of the spectrum of $U\left(T_{0}, 0\right)$ are described in Section 2.3 below. In particular, as a direct consequence of Theorem 2.6. Proposition 2.7 and the fact that $\mu=1$ is an eigenvalue of $U\left(T_{0}, 0\right)$, we obtain the following result.

Proposition 2.5. Let $p_{0}(t)$ be a periodic orbit of (1.1) of period $T_{0}$. Then, $\mu=1$ is an eigenvalue of the period map $U\left(T_{0}, 0\right)$ of multiplicity at most 2 and there is no other eigenvalue on the unit circle. As a consequence, we have the equivalence

$$
p_{0}(t) \text { is hyperbolic } \Leftrightarrow p_{0}(t) \text { is simple. }
$$

Nota Bene. For more general equations than (1.1), if for example, $\mu=\exp 2 i \pi / n$ is an eigenvalue of $U\left(T_{0}, 0\right)$, the periodic orbit $p_{0}(t)$, considered as periodic solution of period $n T_{0}$, will not be simple. So, in general, the definition of simplicity can depend on the chosen period, whereas the definition of the hyperbolicity does not depend on the chosen period. This will not be the case here. Indeed, let $p_{0}(t)$ be a periodic solution of (1.1) with least period $T_{0}$. Proposition 2.5 implies that the generalized eigenfunctions associated to the eigenvalue $\mu=1$ of the map $U\left(n T_{0}, 0\right)$ are exactly the eigenfunctions associated to the eigenvalue $\mu=1$ of the period map $U\left(T_{0}, 0\right)$.

2.3. Spectrum of a general linear operator. In this section, we consider a more general linear equation. Let $T>0$ be any positive time. Let $a$ and $b$ be two functions in $\mathcal{C}^{1}\left(S^{1} \times[0, T], \mathbb{R}\right)$. We consider the operator $U(T, 0): H^{s}\left(S^{1}\right) \longrightarrow$ $H^{s}\left(S^{1}\right)$ defined by $U(T, 0) w_{0}=w(T)$, where $w(t)$ is the solution of

$$
\begin{aligned}
\partial_{t} w(x, t) & =\partial_{x x}^{2} w(x, t)+a(x, t) w(x, t)+b(x, t) \partial_{x} w(x, t), \quad \forall(x, t) \in S^{1} \times(0, T], \\
w(x, 0) & =w_{0}(x) .
\end{aligned}
$$

Due to the classical smoothing properties of parabolic equations, $U(T, 0)$ is a compact operator from $H^{s}\left(S^{1}\right)$ into itself. Thus, the spectrum consists in $\{0\}$ and a sequence of non-zero eigenvalues of finite multiplicity converging to 0 . Notice that 0 is not an eigenvalue, due to the backward uniqueness property of the parabolic equation. We denote by $\left(\lambda_{k}\right)_{k \in \mathbb{N}}$ the eigenvalues of $U(T, 0)$ with the convention that they are repeated according to their multiplicity and ordered by $\left|\lambda_{k+1}\right| \leq\left|\lambda_{k}\right|$.

Using the properties of the zero number, Angenent (2]) has shown the following result (a first statement with $a$ and $b$ analytic was proved in [3]).

Theorem 2.6. Let $\left(\lambda_{k}\right)_{k \in \mathbb{N}}$ be the spectrum of $U(T, 0)$ as introduced above.

Then, for all $j \geq 0,\left|\lambda_{2 j}\right|>\left|\lambda_{2 j+1}\right|$. In particular, $\lambda_{0}$ is a simple real eigenvalue.

Moreover, let $E_{0}$ denote the one-dimensional eigenspace corresponding to $\lambda_{0}$, and, for $j \geq 1$, let $E_{2 j}$ denote the two-dimensional real generalized eigenspace corresponding to $\left\{\lambda_{2 j-1}, \lambda_{2 j}\right\}$. Then, for all $j \geq 0$, any non-zero real function $v \in E_{2 j}$ has exactly $2 j$ zeros, and all these zeros are simple. 
Assume that $\lambda$ is a real eigenvalue and that $\mu$ is another eigenvalue such that $|\lambda|=|\mu|$. Since $a$ and $b$ are real functions, $\bar{\mu}$ is also an eigenvalue. Theorem 2.6 shows that there are at most two eigenvalues with the same modulus, thus $\mu$ is also real. It could be possible that $\mu=-\lambda$, but the following result prevents this case.

Proposition 2.7. Let $j \geq 1$. If $\lambda_{2 j-1}$ and $\lambda_{2 j}$ are two consecutive eigenvalues of $U(T, 0)$, then $\lambda_{2 j-1} \lambda_{2 j}>0$. In particular, if $\lambda$ is a real eigenvalue, then $-\lambda$ is not an eigenvalue and there is no other distinct eigenvalue on the circle $\{z \in \mathbb{C},|z|=$ $|\lambda|\}$.

Proof. Due to Theorem 2.6, there are at most two eigenvalues with the same modulus (counting multiplicity). Since the spectrum is symmetric with respect to the imaginary axis, if $\lambda_{2 j-1}$ or $\lambda_{2 j}$ is not real, then both must be conjugated and $\lambda_{2 j-1} \lambda_{2 j}=\left|\lambda_{2 j}\right|^{2}>0$. For the same reason, it is also clear that the last assertion of Proposition 2.7 is a direct consequence of the first one.

The only case, which has to be studied, is the case when $\lambda_{2 j-1}$ and $\lambda_{2 j}$ are both real. We argue by contradiction: assume that $\lambda_{2 j-1}$ and $\lambda_{2 j}$ are real eigenvalues of $U(T, 0)$ of opposite signs. We denote by $U_{\varepsilon}(T, 0)$ the evolution operator corresponding to

$\partial_{t} w(x, t)=\partial_{x x}^{2} w(x, t)+\varepsilon a(x, t) w(x, t)+\varepsilon b(x, t) \partial_{x} w(x, t), \quad \forall(x, t) \in S^{1} \times(0, T]$.

We denote by $\left(\lambda_{k}^{\varepsilon}\right)_{k \in \mathbb{N}}$ the set of eigenvalues of $U_{\varepsilon}(T, 0)$. We set

$$
\mathcal{E}=\left\{\varepsilon \in[0,1] \mid \lambda_{2 j-1}^{\varepsilon} \text { and } \lambda_{2 j}^{\varepsilon} \text { are real eigenvalues of } U_{\varepsilon}(T, 0) \text { of opposite signs }\right\}
$$

By assumption, $1 \in \mathcal{E}$, and trivially $0 \notin \mathcal{E}$. We will obtain a contradiction by showing that $\mathcal{E}$ is open and closed.

Openness: Let $\varepsilon_{0} \in \mathcal{E}$. Since $\lambda_{2 j}^{\varepsilon_{0}}$ and $\lambda_{2 j-1}^{\varepsilon_{0}}$ are different, they must be simple due to Theorem 2.6. Let $\varphi_{2 j}^{\varepsilon_{0}}$ and $\varphi_{2 j-1}^{\varepsilon_{0}}$ be two corresponding normalized real eigenfunctions. They have $2 j$ simple zeros. The simplicity of the eigenvalues and the implicit function theorem imply that there exists a neighborhood $\mathcal{V}$ of $\varepsilon_{0}$ and functions $\mu_{1}(\varepsilon), \mu_{2}(\varepsilon), \psi_{1}(\varepsilon)$ and $\psi_{2}(\varepsilon)$ defined on $\mathcal{V}$ such that the following properties hold. The functions $\mu_{i}$ are of $\operatorname{class} \mathcal{C}^{0}(\mathcal{V}, \mathbb{R}), \mu_{1}\left(\varepsilon_{0}\right)=\lambda_{2 j-1}^{\varepsilon_{0}}, \mu_{2}\left(\varepsilon_{0}\right)=\lambda_{2 j}^{\varepsilon_{0}}$, and $\mu_{i}(\varepsilon)$ is a simple real eigenvalue of $U_{\varepsilon}(T, 0)$. The functions $\psi_{i}$ are of class $\mathcal{C}^{0}\left(\mathcal{V}, H^{s}\left(S^{1}\right)\right)$, $\psi_{1}\left(\varepsilon_{0}\right)=\varphi_{2 j-1}^{\varepsilon_{0}}, \psi_{2}\left(\varepsilon_{0}\right)=\varphi_{2 j}^{\varepsilon_{0}}$, and $\psi_{i}(\varepsilon)$ is the normalized real eigenfunction corresponding to $\mu_{i}(\varepsilon)$. Since the zeros of all eigenfunctions of $U_{\varepsilon}(T, 0)$ are simple and $H^{s}\left(S^{1}\right) \subset \mathcal{C}^{1}\left(S^{1}\right)$, restricting $\mathcal{V}$ if necessary, we can assume that $\psi_{i}(\varepsilon)$ has exactly $2 j$ zeros which are all simple. Due to Theorem 2.6. this shows that for all $\varepsilon \in \mathcal{V}$, the eigenvalues $\mu_{i}(\varepsilon)$ are the eigenvalues $\lambda_{2 j-1}^{\varepsilon}$ and $\lambda_{2 j}^{\varepsilon}$ of $U_{\varepsilon}(T, 0)$. Moreover, the eigenvalues of $U_{\varepsilon}(T, 0)$ cannot be zero, due to the backward uniqueness property of (2.6). Thus, up to a restriction of $\mathcal{V}$, the eigenvalues $\mu_{i}(\varepsilon)$ are of opposite signs and $\mathcal{V} \subset \mathcal{E}$.

Closeness: Assume that $\left(\varepsilon_{n}\right) \subset \mathcal{E}$ is a given sequence such that $\varepsilon_{n} \longrightarrow \varepsilon$. We first consider the sequence of eigenvalues $\lambda_{2 j}^{\varepsilon_{n}}$ and the sequence of corresponding real eigenfunctions $\varphi_{2 j}^{\varepsilon_{n}}$ with $\left\|\varphi_{2 j}^{\varepsilon_{n}}\right\|_{L^{2}\left(S^{1}\right)}=1$. Taking the inner product in $L^{2}$ of (2.6) with $w$, integrating in time and applying the Gronwall Lemma, we show that there exists a positive constant $C_{0}$ independent of $\varepsilon$ such that every solution $w$ of 
(2.6) satisfies

$$
\|w(T)\|_{L^{2}\left(S^{1}\right)} \leq C_{0}\|w(0)\|_{L^{2}\left(S^{1}\right)} .
$$

Thus, $\left|\lambda_{2 j}^{\varepsilon_{n}}\right|$ is bounded by $C_{0}$ and, up to the extraction of a subsequence, we can assume that $\lambda_{2 j}^{\varepsilon_{n}}$ converges to $\mu$. First, $\mu$ cannot be zero. Indeed, there exists a closed curve $\Gamma \subset \mathbb{C} \backslash\{0\}$ surrounding the $2 j+2$ first eigenvalues of $U_{\varepsilon}(T, 0)$ but not zero. The projector

$$
P_{2 j+2}=-\frac{1}{2 i \pi} \int_{\Gamma}\left(U_{\varepsilon}(T, 0)-z\right)^{-1} d z
$$

is the spectral projector of $U_{\varepsilon}(T, 0)$ onto the space generated by the first $2 j+2$ eigenvalues. Thus, for $n$ large enough,

$$
P_{2 j+2}^{n}=-\frac{1}{2 i \pi} \int_{\Gamma}\left(U_{\varepsilon_{n}}(T, 0)-z\right)^{-1} d z
$$

is a spectral projector of $\operatorname{rank} 2 j+2$, and thus $\Gamma$ still surrounds $2 j+2$ eigenvalues of $U_{\varepsilon_{n}}(T, 0)$. Due to the ordering $\left|\lambda_{0}\right| \geq\left|\lambda_{1}\right| \geq \ldots$, this shows that $\lambda_{2 j}^{\varepsilon_{n}}$ must be away from zero for large $n$. Next, using the smoothing property of parabolic equations shows that there exists a positive constant constant $C_{1}$ independent of $\varepsilon$ such that every solution $w$ of (2.6) satisfies

$$
\|w(T)\|_{H^{2}\left(S^{1}\right)} \leq C_{1}\|w(0)\|_{L^{2}\left(S^{1}\right)} .
$$

Therefore,

$$
\left|\lambda_{2 j}^{\varepsilon_{n}}\right|\left\|\varphi_{2 j}^{\varepsilon_{n}}\right\|_{H^{2}\left(S^{1}\right)} \leq C_{1}\left\|\varphi_{2 j}^{\varepsilon_{n}}\right\|_{L^{2}\left(S^{1}\right)}=C_{1}
$$

Since $\lambda_{2 j}^{\varepsilon_{n}}$ is bounded away from zero, $\left(\varphi_{2 j}^{\varepsilon_{n}}\right)$ is bounded in $H^{2}\left(S^{1}\right)$, and we can assume that $\left(\varphi_{2 j}^{\varepsilon_{n}}\right)$ converges to a function $\psi$ in $\mathcal{C}^{1}\left(S^{1}\right)$. Passing to the limit, we see that $\mu$ is a real eigenvalue of $U_{\varepsilon}(T, 0)$ with normalized eigenfunction $\psi$. Due to Theorem 2.6, $\psi$ has simple zeros only, and since $\left(\varphi_{2 j}^{\varepsilon_{n}}\right)$ converges to $\psi$ in $\mathcal{C}^{1}\left(S^{1}\right)$, the number of zeros of $\psi$ must be $2 j$. Hence, $\mu$ must be equal either to $\lambda_{2 j}^{\varepsilon}$ or to $\lambda_{2 j-1}^{\varepsilon}$. Arguing in the same way for $\lambda_{2 j-1}^{\varepsilon_{n}}$ we show that, up to the extraction of a subsequence, $\lambda_{2 j}^{\varepsilon_{n}}$ and $\lambda_{2 j-1}^{\varepsilon_{n}}$ converge to $\lambda_{2 j}^{\varepsilon}$ or $\lambda_{2 j-1}^{\varepsilon}$. Since neither $\lambda_{2 j}^{\varepsilon}$ nor $\lambda_{2 j-1}^{\varepsilon}$ can be zero and since $\lambda_{2 j}^{\varepsilon_{n}} \lambda_{2 j-1}^{\varepsilon_{n}}<0$, both $\lambda_{2 j}^{\varepsilon}$ and $\lambda_{2 j-1}^{\varepsilon}$ are limits of one of the sequences $\left(\lambda_{2 j}^{\varepsilon_{n}}\right)$ or $\left(\lambda_{2 j-1}^{\varepsilon_{n}}\right)$ and thus are real and of opposite signs. Therefore, $\varepsilon$ belongs to $\mathcal{E}$.

All these properties yield a contradiction since $[0,1]$ is connected. The proposition is proved.

\section{Generic hyperbolicity of the Equilibrium points}

3.1. Generic simplicity. Generic simplicity of equilibria has been proved for various parabolic systems, including the Navier-Stokes equations, in different settings (see [27, 28, 30], 4], 6], 7] for example). Since the proof is rather simple, we include it here for the sake of completeneness. We follow the lines of the proof given by [7] in the case where $f$ does not depend on the derivative $u_{x}$.

Proposition 3.1. For any $n \geq 1$, the set $\mathcal{O}_{n}^{\text {simp }}=\left\{f \in \mathfrak{G} \mid\right.$ any equilibrium point e of (1.1) with $\|e\|_{\mathcal{C}^{1}\left(S^{1}\right)} \leq n$ is simple $\}$

is a dense open subset of $\mathfrak{G}$. As a consequence, the set

$$
\mathcal{O}^{\text {simp }}=\{f \in \mathfrak{G} \mid \text { any equilibrium point e of (1.1) is simple }\}
$$

is a generic subset of $\mathfrak{G}$. 
Proof. If $\mathcal{O}_{n}^{\text {simp }}$ is a dense open set, then, since any equilibrium belongs to $\mathcal{C}^{1}\left(S^{1}\right)$, $\mathcal{O}^{\text {simp }}=\bigcap_{n} \mathcal{O}_{n}^{\text {simp }}$, and thus $\mathcal{O}^{\text {simp }}$ is a generic subset of $\mathfrak{G}$.

Let $n$ be any positive integer.

$\underline{\mathcal{O}}_{n}^{\text {simp }}$ is open: We denote by $R: g \in \mathfrak{G} \mapsto R g \in C^{2}\left(S^{1} \times[-(n+2), n+2] \times\right.$ $[-(n+2), n+2], \mathbb{R})$ the restriction operator defined by

$$
R g=g_{\left[S^{1} \times[-(n+2), n+2] \times[-(n+2), n+2]\right.} .
$$

We notice that $R$ is a continuous, surjective map and that, on $R \mathfrak{G}=C^{2}\left(S^{1} \times[-(n+\right.$ $2), n+2] \times[-(n+2), n+2], \mathbb{R})$, the Whitney topology and the classical $C^{2}$-topology coincide. Since $\mathcal{O}_{n}^{\text {simp }}$ only depends on the values of $f$ in $S^{1} \times[-n, n] \times[-n, n]$, it suffices to show that $R \mathcal{O}_{n}^{\text {simp }}$ is open in $R \mathfrak{G}$ for the classical $C^{2}$-topology. It thus suffices to prove, that if $\left(f_{k}\right)$ is a sequence of functions in $\mathfrak{G} \backslash \mathcal{O}_{n}^{\text {simp }}$ converging to $f$ in $C^{2}\left(S^{1} \times[-(n+2), n+2] \times[-(n+2), n+2], \mathbb{R}\right)$, then $f$ belongs to $\mathfrak{G} \backslash \mathcal{O}_{n}^{\text {simp }}$. Assume that $\left(f_{k}\right)$ is such a sequence of functions in $\mathfrak{G} \backslash \mathcal{O}_{n}^{\text {simp }}$ converging to $f$. Due to Lemma 2.2, there exist a sequence of equilibria $\left(e_{k}\right)$ with $\left\|e_{k}\right\|_{\mathcal{C}^{1}} \leq n$ and a sequence of functions $\left(\varphi_{k}\right) \subset H^{2}\left(S^{1}\right)$ with $\left\|\varphi_{k}\right\|_{L^{2}}=1$ and $L_{e_{k}} \varphi_{k}=0$. Since

$$
\int\left|\partial_{x} \varphi_{k}\right|^{2}=\int D_{u} f_{k}\left(x, e_{k}, \partial_{x} e_{k}\right)\left|\varphi_{k}\right|^{2}+\int D_{u_{x}} f_{k}\left(x, e_{k}, \partial_{x} e_{k}\right) \partial_{x} \varphi_{k} \bar{\varphi}_{k}
$$

the sequence $\left(\varphi_{k}\right)$ is bounded in $H^{1}\left(S^{1}\right)$. Moreover, $\partial_{x x}^{2} e_{k}=f_{k}\left(x, e_{k}, \partial_{x} e_{k}\right)$, and thus the sequence $\left(e_{k}\right)$ is bounded in $H^{s+2}\left(S^{1}\right)$. Up to the extraction of a subsequence, we can assume that $\left(e_{k}\right)$ converges in $H^{2}\left(S^{1}\right)$ to $e$ and $\left(\varphi_{k}\right)$ converges in $\mathbb{L}^{2}\left(S^{1}\right)$ to $\varphi$. Passing to the limit, we obtain $L_{e} \varphi=0$ and $\|e\|_{\mathcal{C}^{1}} \leq n$. By elliptic estimates, $\varphi \in H^{2}\left(S^{1}\right)$; thus $f \notin \mathcal{O}_{n}^{\text {simp }}$, and so $\mathcal{O}_{n}^{\text {simp }}$ is open.

$\underline{\mathcal{O}}_{n}^{\text {simp }}$ is dense: Let $f \in \mathfrak{G}$ be given. We now follow the classical method (see 7] for example). We introduce a function $\chi \in \mathcal{C}^{2}\left(\mathbb{R}^{2}, \mathbb{R}\right)$ satisfying $\chi \equiv 1$ in $[-n-1, n+1]^{2}$ and $\chi \equiv 0$ in $\mathbb{R} \backslash[-n-2, n+2]^{2}$. For any open neighborhood $\mathcal{V}$ of $f$ in $\mathfrak{G}$, there exists an open neighborhood $\mathcal{U}$ of 0 in $\mathcal{C}^{2}\left(S^{1}\right)$ such that, for all $a \in \mathcal{U}$, the function $(x, u, v) \mapsto f(x, u, v)+a(x) \chi(u, v)$ belongs to $\mathcal{V}$. So, it is sufficient to prove that there is a dense set of functions $a \in \mathcal{C}^{2}\left(S^{1}, \mathbb{R}\right)$ such that $f+a \chi \in \mathcal{O}_{n}^{\text {simp }}$. To obtain this density, we apply the Sard-Smale Theorem (Theorem A.1 in the appendix) to the functional $\Phi:(e, a) \in\left\{e \in H^{2}\left(S^{1}\right),\|e\|_{\mathcal{C}^{1}}<n+1\right\} \times \mathcal{C}^{2}\left(S^{1}, \mathbb{R}\right) \mapsto$ $\Phi(e, a) \in L^{2}\left(S^{1}\right)$ defined by

$$
\Phi(e, a)=e_{x x}+f\left(x, e, e_{x}\right)+a(x) \chi\left(e, e_{x}\right)
$$

and to the point $z=0$.

Assumption iii) of Theorem A.1 is satisfied. To simplify the notation, let us prove i) and ii) with $a=0$, which does not lead to any loss of generality. First notice that $(e, 0) \in \Phi^{-1}(0)$ means that $e$ is an equilibrium point of (1.1). Assumption i) of Theorem A.1 is satisfied since $D_{e} \Phi(e, 0)=L_{e}$ is a Fredholm operator of index 0 due to Lemma 2.2. To prove ii), we must find for each $h \in L^{2}\left(S^{1}\right)$ a pair $(\varphi, b)$ such that $D_{e, a} \Phi(e, 0) \cdot(\varphi, b)=L_{e} \varphi+b(x) \chi\left(e, e_{x}\right)=L_{e} \varphi+b(x)=h$. Since $L_{e}$ is Fredholm, $h-$ $b \in \operatorname{Im}\left(L_{e}\right)$ if and only if, for all $\psi \in \operatorname{Ker}\left(L_{e}^{*}\right), \int(h-b) \psi=0$. As $\operatorname{Ker}\left(L_{e}^{*}\right)$ is finitedimensional, we can introduce a finite orthonormal basis $\left(\psi_{i}\right)_{i=1, \ldots, p}$ of $\operatorname{Ker}\left(L_{e}^{*}\right)$. We are reduced to finding $b$ such that $\int(h-b) \psi_{i}=0$ for all $i=1, \ldots, p$, that is, so that 0 belongs to the image of the map: $b \in \mathcal{C}^{2}\left(S^{1}\right) \longmapsto\left(\int(h-b) \psi_{i}\right)_{i=1, \ldots, p}$. 
This image is closed since it is an affine subspace of $\mathbb{R}^{p}$, and by density of $\mathcal{C}^{2}$ in $L^{2}$ we can find $b$ as close to $h$ as is wanted. Therefore, we can find $b$ such that $\int(h-b) \psi_{i}=0$ for all $i=1, \ldots, p$, and thus $D_{e, a} \Phi$ is surjective. Actually, remarking that the vectors $\psi_{i}$ belong to $H^{3}\left(S^{1}\right)$, we can simply choose $b=\sum_{0}^{p}\left(h, \psi_{i}\right) \psi_{i}$.

The conclusion of Theorem A.1 shows that for a generic $a \in \mathcal{C}^{2}\left(S^{1}\right)$, any $e$ satisfying $\|e\|_{\mathcal{C}^{1}} \leq n$ and $e_{x x}+f\left(x, e, e_{x}\right)+a(x) \chi\left(e, e_{x}\right)=0$ is such that $D_{e} \Phi=L_{e}$ is surjective, which implies by Lemma 2.2 that $e$ is a simple equilibrium point. Thus, we can choose a function $a$ as small as is wanted such that $f+a \chi \in \mathcal{O}_{n}^{\text {simp }}$, which shows that $\mathcal{O}_{n}^{\text {simp }}$ is dense.

3.2. Generic hyperbolicity. In Proposition 3.1, we have proved the generic simplicity of the equilibrium points of (1.1). Using this result, it is not difficult to show the generic hyperbolicity.

Proposition 3.2. For any $n \geq 1$, the set

$$
\mathcal{O}_{n}^{h}=\left\{f \in \mathfrak{G} \mid \text { any equilibrium point } e \text { of (1.1) with }\|e\|_{\mathcal{C}^{1}\left(S^{1}\right)} \leq n \text { is hyperbolic }\right\}
$$

is a dense open subset of $\mathfrak{G}$. As a consequence, the set

$$
\mathcal{O}^{h}=\{f \in \mathfrak{G} \mid \text { any equilibrium point } e \text { of (1.1) is hyperbolic }\}
$$

is a generic subset of $\mathfrak{G}$.

Proof. As in the proof of Proposition 3.1, it is sufficient to show that $\mathcal{O}_{n}^{\mathrm{h}}$ is a dense open set.

$\underline{\mathcal{O}}_{n}^{\mathrm{h}}$ is open: As in the proof of Proposition 3.1 it is sufficient to prove that if $\left(f_{k}\right)$ is a sequence of functions in $\mathfrak{G} \backslash \mathcal{O}_{n}^{h}$ converging to $f$ in $C^{2}\left(S^{1} \times[-(n+2), n+2] \times\right.$ $[-(n+2), n+2], \mathbb{R})$, then $f$ belongs to $\mathfrak{G} \backslash \mathcal{O}_{n}^{h}$. So let $\left(f_{k}\right)$ be such a sequence of functions in $\mathfrak{G} \backslash \mathcal{O}_{n}^{h}$ converging to $f$. Then there exist sequences $\left(e_{k}\right) \subset H^{s+2}\left(S^{1}\right)$, $\left(\lambda_{k}\right) \subset i \mathbb{R}$ and $\left(\varphi_{k}\right) \subset H^{2}\left(S^{1}\right)$ such that $\partial_{x x}^{2} e_{k}+f_{k}\left(x, e_{k}, \partial_{x} e_{k}\right)=0,\left\|e_{k}\right\|_{\mathcal{C}^{1}} \leq n$, $\left\|\varphi_{k}\right\|_{L^{2}}=1$ and $L_{e_{k}} \varphi_{k}=\lambda_{k} \varphi_{k}$. As in the proof of Proposition 3.1, we can assume that $\left(e_{k}\right)$ converges in $H^{2}\left(S^{1}\right)$ to a function $e$. Then, the proof of openness will be exactly the same as the corresponding one in Proposition 3.1 as soon as we show that there exists a subsequence $\left(\lambda_{k_{n}}\right) \subset i \mathbb{R}$, which is convergent. This is a consequence of Estimate (2.3).

$\underline{\mathcal{O}}_{n}^{\mathrm{h}}$ is dense: Let $f_{0} \in \mathfrak{G}$. We are going to show that we can construct successive perturbations of $f_{0}$, as small as needed, to obtain a function $f \in \mathcal{O}_{n}^{\mathrm{h}}$ as close to $f_{0}$ as is wanted. First, due to Proposition 3.1, we can find $f_{1}$ close to $f_{0}$ such that all equilibrium points of $f_{1}$ are simple. The set $\left\{e \mid \partial_{x x}^{2} e+f_{1}\left(x, e, e_{x}\right)=0,\|e\|_{\mathcal{C}^{1}} \leq\right.$ $n+1\}$ is bounded in $H^{s+2}\left(S^{1}\right)$ and hence compact in $H^{2}\left(S^{1}\right)$. Since all equilibrium points of $f_{1}$ are simple and thus isolated, there is a finite number of equilibria $e_{1}, \ldots, e_{p}$ of $f_{1}$ which satisfy $\left\|e_{j}\right\|_{\mathcal{C}^{1}} \leq n+1$.

We next explain how each equilibrium $e_{i}$ can be made hyperbolic by successive perturbations of $f_{1}$. Let $e$ be an equilibrium point of (1.1) with $\|e\|_{\mathcal{C}^{1}} \leq n$, and let us denote $\left(\lambda_{k}\right)_{k \in \mathbb{N}}$ the sequence of eigenvalues of the corresponding linearized operator $L_{e}$. Let $\chi \in \mathcal{C}^{2}(\mathbb{R}, \mathbb{R})$ be a smooth cut-off function such that, for example, $\chi(y)=y$ for $|y| \leq n+1$ and $\chi(y)=0$ for $|y| \geq n+2$. Then, $\chi(e(x))=e(x)$ for any $x \in S^{1}$. If we perturb $f_{1}$ by setting for small $\alpha \in \mathbb{R}, f_{\alpha}(x, v, w)=f_{1}(x, v, w)+\alpha(\chi(v)-e(x))$, then $e$ is still an equilibrium point of (1.1) with $f$ replaced by $f_{\alpha}$ and the spectrum of $L_{e}$ becomes $\left(\lambda_{k}+\alpha\right)_{k \in \mathbb{N}}$. Since the eigenvalues of $L_{e}$ are isolated, this means that one can perturb $f_{1}$ such that $L_{e}$ no longer has eigenvalues on the imaginary 
axis, that is, such that $e$ becomes hyperbolic. On the other hand, by the implicit functions theorem, if $e$ is a simple (resp. a hyperbolic) equilibrium of $f$, there exist neighborhoods $\mathcal{V} \subset \mathcal{C}^{1}\left(S^{1}\right)$ of $e$ and $\mathcal{U} \subset \mathfrak{G}$ of $f$ such that, for all $g \in \mathcal{U}$, there exists a unique equilibrium point $e(g) \in \mathcal{V}$ and that this equilibrium is simple (resp. hyperbolic). Thus, we can successively make each equilibrium hyperbolic without changing the status of the other equilibria $e_{1}, \ldots, e_{p}$.

\section{Generic hyperbolicity of the Periodic orbits}

The aim of this section is the proof of the genericity theorem, Theorem 1.2 , That is, we want to show that there exists a subset $\mathcal{O}$ of $\mathfrak{G}$ such that, for all $f \in \mathcal{O}$, all the equilibrium points and all the periodic solutions of (1.1) are hyperbolic.

To show Theorem 1.2, we use the induction argument of Peixoto [26] in the case of vector fields on compact manifolds or of Mallet-Paret [21] in the case of functional differential equations. Thus, for any $n \geq 1$ and any $A>0$, we introduce the set

$$
\begin{aligned}
\mathcal{O}(A, n)= & \left\{f \in \mathcal{O}_{n}^{h} \mid \text { all non-constant periodic orbits } p(t)\right. \text { of (1.1) with period } \\
& \left.T \in(0, A] \text { such that } \sup _{t \in \mathbb{R}}\|p(t)\|_{C^{1}\left(S^{1}\right)} \leq n \text { are non-degenerate }\right\} .
\end{aligned}
$$

Due to Proposition 2.5, we have the following equality:

$$
\begin{aligned}
\mathcal{O}(A, n)= & \left\{f \in \mathcal{O}_{n}^{h} \mid \text { all non-constant periodic orbits } p(t)\right. \text { of (1.1) with period } \\
& \left.T \in(0, A] \text { such that } \sup _{t \in \mathbb{R}}\|p(t)\|_{C^{1}\left(S^{1}\right)} \leq n \text { are hyperbolic }\right\} .
\end{aligned}
$$

As in [26] and in 21], we show that $\mathcal{O}(n, n)$ is open and dense in $\mathfrak{G}$. Since $\mathfrak{G}$ is a Baire space, it follows that $\mathcal{O}=\bigcap_{n=1}^{\infty} \mathcal{O}(n, n)$ is a generic subset and hence a dense subset of $\mathfrak{G}$, which proves Theorem 1.2 ,

Let $n \in \mathbb{N}$ be fixed for the remaining part of the section. We first begin with auxiliary results, which will be widely used in this section. In particular, we prove that, for any $A>0, \mathcal{O}(A, n)$ is open. To simplify our statements below, we denote by $S_{f}(t)$ the local nonlinear semigroup defined by the equation (1.1) with nonlinearity $f$.

Proposition 4.1. The following properties hold.

(a) Let $f \in \mathcal{O}_{n}^{\text {simp }}$ (resp. $f \in \mathcal{O}_{n}^{h}$ ). There exists $\delta>0$ such that $f \in \mathcal{O}_{n+\delta}^{\text {simp }}$ (resp. $\left.f \in \mathcal{O}_{n+\delta}^{h}\right)$.

(b) For any $\mu>0$, the set $\mathcal{O}(A, \mu)$ is open.

(c) Let $f \in \mathcal{O}_{n}^{\text {simp }}$ and let $\delta$ be as in (a). There exist $\varepsilon>0$ and a neighborhood $\mathcal{N}_{1} \subset \mathcal{O}_{n+\delta}^{\text {simp }}$ of $f$ such that, for any $g \in \mathcal{N}_{1}$, any nonconstant periodic solution $p(t)$ of $S_{g}(t)$ with $\sup _{t \in \mathbb{R}}\|p(t)\|_{C^{1}\left(S^{1}\right)} \leq n+\delta$ has a smallest period strictly larger than $\varepsilon$.

(d) Let $f \in \mathcal{O}_{n}^{h}$ and let $\delta$ be as in (a). For any $A>0$, there exist a positive constant $r$ and a neighborhood $\mathcal{N}_{2} \subset \mathcal{O}_{n+\delta}^{h}$ of $f$ such that the following property holds. Let $\mathcal{E}_{f, n+\delta}$ be the set of the equilibrium points $e_{f}$ of $S_{f}(t)$ satisfying $\left\|e_{f}\right\|_{C^{1}\left(S^{1}\right)} \leq n+\delta$ and let $\mathcal{B E}(f, n+\delta, r)=\cup_{e_{f} \in \mathcal{E}_{f, n+\delta}} B_{H^{s}\left(S^{1}\right)}\left(e_{f}, r\right)$. For any $g \in \mathcal{N}_{2}, \mathcal{E}_{g, n+\delta} \subset \mathcal{B E}(f, n+\delta, r)$ and the set of all nonconstant periodic orbits $p(t)$ of $S_{g}(t)$ of period less than $A$ and satisfying $\sup _{t \in \mathbb{R}}\|p(t)\|_{C^{1}\left(S^{!}\right)} \leq n+\delta$ does not intersect $\mathcal{B E}(f, n+\delta, r)$.

Proof. The proof of property (a) is similar to the proof of the openness of $\mathcal{O}_{n}^{\text {simp }}$ in Proposition 3.1 
To prove property (b), we follow the lines of the proof of Theorem 2.1 of 21]. By the remarks made at the beginning of the proof of Proposition 3.1, it is sufficient to prove that, if $f_{m}$ is a sequence of functions in $\mathfrak{G} \backslash \mathcal{O}(A, \mu)$ converging to $f$ in $C^{2}\left(S^{1} \times[-(\mu+2), \mu+2] \times[-(\mu+2), \mu+2], \mathbb{R}\right)$, then $f$ belongs to $\mathfrak{G} \backslash \mathcal{O}(A, \mu)$. So assume that $f_{m}$ is such a sequence of functions in $\mathfrak{G} \backslash \mathcal{O}(A, \mu)$ converging to $f$ in $C^{2}\left(S^{1} \times[-(\mu+2), \mu+2] \times[-(\mu+2), \mu+2], \mathbb{R}\right)$. Then, for any $m$, there exists a non-simple periodic solution $p_{m}(t)$ of

$$
u_{t}(x, t)=u_{x x}(x, t)+f_{m}\left(x, u, u_{x}\right), \quad(x, t) \in S^{1} \times \mathbb{R}_{+}^{*},
$$

with a non-simple period $T_{m} \in(0, A)$. Moreover, $p_{m}(t)$ satisfies the following bound:

$$
\sup _{t}\left\|p_{m}(t)\right\|_{C^{1}\left(S^{1}\right)} \leq \mu .
$$

Due to the smoothness hypotheses made on $f$ and to the bound (4.2), we can show by a recursion argument that the function $p_{m}(t)$ belongs to $C^{1}\left([0,2 A], H^{s}\left(S^{1}\right)\right) \cap$ $C^{0}\left([0,2 A], H^{2}\left(S^{1}\right)\right)$ and that there exists a positive constant $C_{0}$ such that, for any $m \in \mathbb{N}$,

$$
\left\|p_{m}(t)\right\|_{C^{1}\left([0,2 A], H^{s}\left(S^{1}\right)\right) \cap C^{0}\left([0,2 A], H^{2}\left(S^{1}\right)\right)} \leq C_{0} .
$$

Thus, the family of mappings $p_{m}(t) \in C^{1}\left([0,2 A], H^{s}\left(S^{1}\right)\right), m \in \mathbb{N}$, is equicontinuous from $[0, A]$ into $H^{s}\left(S^{1}\right)$. By the Ascoli theorem, there exists a subsequence $\left(p_{m_{j}}(t)\right)$ which converges to a function $p(t)$ in $C^{0}\left([0,2 A], H^{s}\left(S^{1}\right)\right)$ and where $p(t)$ belongs to $C^{0}\left([0,2 A], H^{s}\left(S^{1}\right)\right)$. Furthermore, $f_{m_{j}}\left(x, p_{m_{j}}(t), \partial_{x} p_{m_{j}}(t)\right)$ converges to $f\left(x, p(t), \partial_{x} p(t)\right)$ in $C^{0}\left([0,2 A], L^{2}\left(S^{1}\right)\right)$. Now taking the limits in the variation of constants formula

$$
p_{m_{j}}(t)=e^{B t} p_{m_{j}}(0)+\int_{0}^{t} e^{B(t-s)}\left(p_{m_{j}}(s)+f_{m_{j}}\left(x, p_{m_{j}}(s), \partial_{x} p_{m_{j}}(s)\right)\right) d s,
$$

where $B$ is the self-adjoint operator $B=\partial_{x x}-I$, we conclude that $p(t)$ is a mild solution of (1.1) on the time interval $[0,2 A]$, that is, of the equation

$$
p(t)=e^{B t} p(0)+\int_{0}^{t} e^{B(t-s)}\left(p(s)+f\left(x, p(s), \partial_{x} p(s)\right)\right) d s .
$$

Since $p_{m}(t)$ is a periodic solution of period $T_{m} \leq A$, we also conclude that $p(t)$ is a periodic solution of (1.1) of period $T^{*}=\lim _{m \rightarrow+\infty} T_{m}$ and that the integral equality (4.4) holds on $\mathbb{R}^{+}$. Furthermore, $p(t)$ is a classical solution of (4.4).

We next consider the linearized equations

$$
\begin{aligned}
\varphi_{t}(x, t) & =\varphi_{x x}(x, t)+D_{u} f_{m}\left(x, p_{m}, \partial_{x} p_{m}\right) \varphi+D_{u_{x}} f_{m}\left(x, p_{m}, \partial_{x} p_{m}\right) \varphi_{x}, \\
\varphi(x, 0) & =\varphi_{0}(x),
\end{aligned}
$$

as well as the associated linear operators $U_{m}(t, 0)$ defined by $U_{m}(t, 0) \varphi_{0}=\varphi^{m}(t)$, where $\varphi^{m}(t)$ is the solution of (4.5). We recall that $U_{m}\left(T_{m}, 0\right): H^{s}\left(S^{1}\right) \mapsto H^{s}\left(S^{1}\right)$ is a compact map. By assumption, the element 1, which belongs to the spectrum $\sigma\left(U_{m}\left(T_{m}, 0\right)\right)$ of $U_{m}\left(T_{m}, 0\right)$, is of multiplicity greater than one.

We also consider the operator $U(t, 0)$ associated to the limiting linearized equation

$$
\begin{aligned}
\varphi_{t}(x, t) & =\varphi_{x x}(x, t)+D_{u} f\left(x, p, \partial_{x} p\right) \varphi+D_{u_{x}} f\left(x, p, \partial_{x} p\right) \varphi_{x} \\
\varphi(x, 0) & =\varphi_{0}(x) .
\end{aligned}
$$


Since $p_{m_{j}}(t)$ converges to a function $p(t)$ in $C^{0}\left([0,2 A], H^{s}\left(S^{1}\right)\right)$ and $f_{m}$ converges to $f$ in $C^{2}\left(S^{1} \times[-(\mu+2), \mu+2] \times[-(\mu+2), \mu+2], \mathbb{R}\right)$, we easily prove that the operator $U_{m_{j}}(t, 0)$ converges to $U(t, 0)$ in $\mathcal{L}\left(H^{s}\left(S^{1}\right), H^{s}\left(S^{1}\right)\right)$, uniformly in $t \in[0,2 A]$. Assume that $T^{*} \neq 0$. Then, $U_{m_{j}}\left(T_{m_{j}}, 0\right)$ converges to $U\left(T^{*}, 0\right)$ in $\mathcal{L}\left(H^{s}\left(S^{1}\right), H^{s}\left(S^{1}\right)\right)$.

Now three cases may arise:

1) The periodic orbit $p(t)$ is non-constant and $T^{*}>0$. The element $1 \in$ $\sigma\left(U\left(T_{m}, 0\right)\right)$ has multiplicity greater than one, so $1 \in \sigma\left(U\left(T^{*}, 0\right)\right)$ also has multiplicity greater than one. Thus $p(t)$ is a non-simple periodic solution with the non-simple period $T^{*}$ and $f \notin \mathcal{O}(A, \mu)$.

2) The periodic orbit $p(t)$ is a constant $a$ and $T^{*}>0$. The element 1 belongs to the spectrum $\sigma\left(U\left(T_{m}, 0\right)\right)$ of $U\left(T_{m}, 0\right)$. Thus, 1 also belongs to the spectrum $\sigma\left(U\left(T^{*}, 0\right)\right)$ of $U\left(T^{*}, 0\right)$. This implies that $a$ is not hyperbolic. Thus, $f \notin \mathcal{O}_{\mu}^{h}$ and $f \notin \mathcal{O}(A, \mu)$.

3) The periodic orbit $p(t)$ is a constant $a$ and $T^{*}=0$. For any $T_{0} \in(0,2 A]$, we can find a sequence of positive integers $N_{m}$ such that $N_{m} T_{m}$ converges to $T_{0}$. Then, $U_{m_{j}}\left(N_{m_{j}} T_{m_{j}}, 0\right)$ converges to $U\left(T_{0}, 0\right)$ in $\mathcal{L}\left(H^{s}\left(S^{1}\right), H^{s}\left(S^{1}\right)\right)$. Thus, 1 belongs to the spectrum $\sigma\left(U\left(T_{0}, 0\right)\right)$ of $U\left(T_{0}, 0\right)$. Since, as explained in Section 2.2, $U\left(T_{0}, 0\right)=$ $e^{L_{a} T_{0}}$ and $L_{a}$ is a sectorial operator, the inclusions (2.4) hold, with $e$ replaced by $a$ and with $t$ replaced by any $T_{0} \in(0,2 A]$. It follows that 0 belongs to the spectrum of $L_{a}$, which means that $a$ is not a simple equilibrium point and $f$ does not belong to the set $\mathcal{O}_{\mu}^{\text {simp }}$, and thus it does not belong to $\mathcal{O}(A, \mu)$.

To prove the assertions (c) and (d), we argue as in the above cases 2) and 3). Proposition 4.1 is proved.

Before entering into the proof of the density of the sets $\mathcal{O}(A, n)$, we want to emphasize that the two main ingredients of this proof are the Sard-Smale Theorem and the properties of the zero number. The properties of the zero number are recalled in the Introduction (for more details, see [31, 2, 22]). These properties are used here through Proposition 2.5 and the following primordial lemma.

Lemma 4.2. Let $p_{0}(t)$ be a periodic solution of (1.1) of least period $T_{0}>0$. The map

$$
(x, t) \in S^{1} \times\left[0, T_{0}\right) \mapsto\left(x, p_{0}(x, t), \partial_{x} p_{0}(x, t)\right)
$$

is one to one.

Proof. Assume that this map is not injective. Then there exist $x_{0}, t_{0} \in\left[0, T_{0}\right)$ and $t_{1} \in\left[0, T_{0}\right), t_{0} \neq t_{1}$ such that

$$
p_{0}\left(x_{0}, t_{0}\right)=p_{0}\left(x_{0}, t_{1}\right), \quad \partial_{x} p_{0}\left(x_{0}, t_{0}\right)=\partial_{x} p_{0}\left(x_{0}, t_{1}\right) .
$$

The function $v(x, t)=p_{0}\left(x, t+t_{1}-t_{0}\right)-p_{0}(x, t)$ is a solution of the equation

$$
v_{t}(x, t)=v_{x x}(x, t)+b(x, t) v(x, t)+d(x, t) v_{x}(x, t),
$$

where $b(x, t)=\int_{0}^{1} D_{u} f\left(x, p_{0}(t)+s\left(p_{0}\left(t+t_{1}-t_{0}\right)-p_{0}(t)\right), \partial_{x} p_{0}\left(t+t_{1}-t_{0}\right)\right) d s$ and $d(x, t)=\int_{0}^{1} D_{u_{x}} f\left(x, p_{0}(t), \partial_{x}\left(p_{0}(t)+s\left(p_{0}\left(t+t_{1}-t_{0}\right)-p_{0}(t)\right)\right)\right) d s$. Moreover, the function $v(x, t)$ satisfies $v\left(x_{0}, t_{0}\right)=0$ and $\partial_{x} v\left(x_{0}, t_{0}\right)=0$, and it does not vanish everywhere since $\left|t_{1}-t_{0}\right|<T_{0}$. Thus, the zero number $z(v(t))$ drops strictly at $t=t_{0}$. Since $v(t)$ is a periodic function of period $T_{0}$, this leads to a contradiction with the fact that $z\left(v\left(t+T_{0}\right)\right)=z(v(t))$. The lemma is proved. 
To prove that $\mathcal{O}(n, n)$ is dense in $\mathfrak{G}$, we argue as follows. Let $f_{0} \in \mathcal{O}_{n}^{h}$. Due to Proposition 4.1 there exists a constant $\delta \in(0,1)$ such that $f_{0} \in \mathcal{O}_{n+\delta}^{h}$. Let $\delta \in(0,1), \varepsilon>0$ and $\mathcal{N}_{1}$ be as in Proposition 4.1 (a) and (c). And for $A=n+2$, let $r>0, \mathcal{B E}\left(f_{0}, n+\delta, r\right)$ and $\mathcal{N}_{2}$ be as in Proposition 4.1 (d). We next choose a neighborhood $\mathcal{N}^{0}=\mathcal{N}_{1} \cap \mathcal{N}_{2}$ of $f_{0}$. Since $\mathcal{O}_{n+\delta}^{h}$ is open, we can also assume that $\mathcal{N}^{0} \subset \mathcal{O}_{n+\delta}^{h}$. Let $\eta$ be a small positive constant such that $\eta \sum_{j=1}^{+\infty} j^{-4} \leq \delta / 2$. We set

$$
\mathcal{P}(k)=\mathcal{O}\left((3 / 2)^{k} \varepsilon, n+\delta-\eta \sum_{j=1}^{k} j^{-4}\right) .
$$

Obviously, one can choose $\varepsilon$ as small as needed so that there exists $k_{0} \in \mathbb{N}$ such that $n \leq(3 / 2)^{k_{0}} \varepsilon<n+2$. In Proposition 4.3 below, we show that for all $k \in \mathbb{N}$, $0<k \leq k_{0}$, there exists an open neighbourhood $\mathcal{N}^{k}, \mathcal{N}^{k} \subset \mathcal{N}^{k-1}$, such that $\mathcal{P}(k) \cap \overline{\mathcal{N}}^{k}$ is dense in $\mathcal{P}(k-1) \cap \mathcal{N}^{k}$. Since $\mathcal{P}(0)=\mathcal{O}(\varepsilon, n+\delta)$, Property (c) of Proposition 4.1 implies that $\mathcal{P}(0) \cap \mathcal{N}^{0}=\mathcal{O}_{n+\delta}^{h} \cap \mathcal{N}^{0}=\mathcal{N}^{0}$. These two properties show by recursion that $\mathcal{P}(k) \cap \mathcal{N}^{k}$ is dense in $\mathcal{N}^{k}$ for any $k$. Hence, $\mathcal{O}(n, n) \cap \mathcal{N}^{k_{0}}$ is dense in $\mathcal{N}^{k_{0}}$. As $f_{0} \in \mathcal{O}_{n}^{h}$ is arbitrary and as $\mathcal{O}_{n}^{h}$ is dense in $\mathfrak{G}$, it follows that $\mathcal{O}(n, n)$ is dense in $\mathfrak{G}$.

Proposition 4.3. For any $k \in \mathbb{N}, 0<k \leq k_{0}$, there exists a neighbourhood $\mathcal{N}^{k} \subset \mathcal{N}^{k-1}$ of $f_{0}$ such that the set $\mathcal{P}(k) \cap \mathcal{N}^{k}$ is dense in $\mathcal{P}(k-1) \cap \mathcal{N}^{k}$.

Proof. We apply Theorem A.1 from the appendix with the following Banach spaces and functional $\Phi$. Let $0<\delta^{*} \leq 1 / 4$, we set:

$$
\begin{aligned}
& U=\left\{\left(T, u_{0}\right) \in\left(\varepsilon,\left((3 / 2)^{k}+\delta^{*}\right) \varepsilon\right) \times H^{s}\left(S^{1}\right) \mid u_{0} \notin \overline{\mathcal{B E}}\left(f_{0}, n+\delta, r\right),\right. \\
& \left.\quad \text { and } \sup _{t \in\left[0,\left((3 / 2)^{k}+\delta^{*}\right) \varepsilon\right]}\left\|S_{f_{0}}(t) u_{0}\right\|_{C^{1}\left(S^{1}\right)}<n+\delta-\eta \sum_{j=1}^{k-1} j^{-4}-\frac{\eta}{2} k^{-4}\right\}, \\
& Z=H^{s}\left(S^{1}\right) .
\end{aligned}
$$

We would like to choose $\mathcal{P}(k-1) \cap \mathcal{N}^{k-1}$ as set $V$. However, the space $\mathfrak{G}$ is not even metrizable. To overcome this problem, one has to work with functions defined on compact sets. We notice that we can choose a neighbourhood $\mathcal{N}^{k} \subset \mathcal{N}^{k-1}$ of $f_{0}$ such that, if

$$
\sup _{t \in\left[0,\left((3 / 2)^{k}+\delta^{*}\right) \varepsilon\right]}\left\|S_{f_{0}}(t) u_{0}\right\|_{C^{1}\left(S^{1}\right)}<n+\delta-\eta \sum_{j=1}^{k-1} j^{-4}-\frac{\eta}{2} k^{-4},
$$

then, for any $g \in \mathcal{N}^{k}$,

$$
\sup _{t \in\left[0,\left((3 / 2)^{k}+\delta^{*}\right) \varepsilon\right]}\left\|S_{g}(t) u_{0}\right\|_{C^{1}\left(S^{1}\right)}<n+\delta-\eta \sum_{j=1}^{k-1} j^{-4}-\frac{\eta}{4} k^{-4}
$$

Let $R: g \in \mathfrak{G} \mapsto R g \in C^{2}\left(S^{1} \times[-(n+2), n+2] \times[-(n+2), n+2], \mathbb{R}\right)$ be the restriction operator defined by

$$
R g=g_{\mid S^{1} \times[-(n+2), n+2] \times[-(n+2), n+2]} .
$$

We set $V=R\left(\mathcal{P}(k-1) \cap \mathcal{N}^{k}\right)$ endowed with the topology of $C^{2}\left(S^{1} \times[-(n+2), n+\right.$ $2] \times[-(n+2), n+2], \mathbb{R})$, which is a separable Banach space. The map $R$ is a continuous, open and surjective map. Therefore the density of $R\left(\mathcal{P}(k) \cap \mathcal{N}^{k}\right)$ in $V$ 
is equivalent to the density of $\mathcal{P}(k) \cap \mathcal{N}^{k}$ in $\mathcal{P}(k-1) \cap \mathcal{N}^{k}$. We set $z=0$, and we consider the functional $\Phi: U \times V \rightarrow Z$ defined by

$$
\Phi:\left(t, u_{0}, f\right) \in U \times V \mapsto \Phi\left(t, u_{0}, f\right)=S_{f}(t) u_{0}-u_{0} .
$$

As it will become clear below, $\Phi$ is a $C^{2}$-map from $U \times V$ into $Z$. Let us make some remarks on the choice of this functional. First, for any $\left(T, u_{0}\right) \in U$, the trajectory $S_{g}(t) u_{0}$ only depends on the value of $R g$. The use of the restriction operator $R$ does not affect any trajectory considered here.

Next, we remark that $\Phi^{-1}(0)=\left\{\left(T, u_{0}, f\right) \in U \times V \mid S_{f}(t) u_{0}\right.$ is a periodic orbit of (1.1) of period $T\}$. For any $(T, p(0), f) \in \Phi^{-1}(0)$, either $T$ is a simple period of the periodic orbit $p(t)=S_{f}(t) p(0)$, or $T$ is not a simple period. Since $f$ belongs to $R\left(\mathcal{P}(k-1) \cap \mathcal{N}^{k}\right)$, any periodic orbit $p(t)$ of (1.1) with period $T \in\left(0,(3 / 2)^{k-1} \varepsilon\right]$ and such that $\sup _{t}\|p(t)\|_{C^{1}\left(S^{1}\right)} \leq n+\delta-\eta \sum_{j=1}^{k-1} j^{-4}$ is hyperbolic. Therefore, as noticed in the remark following Proposition 2.5], if $T$ is not a simple period of $p(t)$, then $T$ must belong to $\left((3 / 2)^{k-1} \varepsilon,\left((3 / 2)^{k}+\delta^{*}\right) \varepsilon\right]$ and must be the minimal period of $p(t)$.

Working with sets involving a sequence of bounds of the type $n+\delta-\eta \sum_{j=1}^{k-1} j^{-4}-$ $\frac{\eta}{2} k^{-4}$ looks complicated and technical. Actually, in the definition of sets as $\mathcal{O}_{\mu}^{h}$ or $\mathcal{O}(A, \mu)$, we need large inequalities (i.e. the symbols $\leq$ ), in order to obtain open sets, whereas the definition of the open set $U$ requires strict inequalities (i. e. symbols $<)$. Thus, working at the same time with large and strict inequalities, requires to introduce some intermediate bounds, which look complicated and artificial.

We now check that the hypotheses of Theorem A.1 are satisfied.

Hypothesis i) of Theorem A.1 holds. Indeed, for any $(T, p(0), f) \in \Phi^{-1}(0)$, we have

$D_{t, u} \Phi(T, p(0), f)(\tau, v)=p_{t}(T) \tau+\left(D_{u}\left(S_{f}(T) p(0)\right)-I\right) v \equiv p_{t}(T) \tau+\left(U_{f, p}(T, 0)-I\right) v$, where the linearized operator $U_{f, p}$ has been introduced in (2.1). Since $U_{f, p}$ : $H^{s}\left(S^{1}\right) \rightarrow H^{s}\left(S^{1}\right)$ is a compact operator, the operator $U_{f, p}(T, 0)-I$ is a Fredholm operator of index 0 . We recall that $\operatorname{dim}\left(\operatorname{Ker}\left(U_{f, p}(T, 0)-I\right)\right) \geq 1$. Now two cases can occur. Either $p_{t}(T)$ belongs to $\operatorname{Im}\left(U_{f, p}(T, 0)-I\right)$ and thus

$$
\operatorname{dim}\left(\operatorname{Ker} D_{t, u} \Phi(T, p(0), f)\right)=\operatorname{dim}\left(\operatorname{Ker}\left(U_{f, p}(T, 0)-I\right)\right)+1
$$

and

$$
\operatorname{codim}\left(\operatorname{Im} D_{t, u} \Phi(T, p(0), f)\right)=\operatorname{codim}\left(\operatorname{Im}\left(U_{f, p}(T, 0)-I\right)\right),
$$

or $p_{t}(T)$ does not belong to $\operatorname{Im}\left(U_{f, p}(T, 0)-I\right)$ and thus

$$
\operatorname{dim}\left(\operatorname{Ker} D_{t, u} \Phi(T, p(0), f)\right)=\operatorname{dim}\left(\operatorname{Ker}\left(U_{f, p}(T, 0)-I\right)\right)
$$

and

$$
\operatorname{codim}\left(\operatorname{Im} D_{t, u} \Phi(T, p(0), f)\right)=\operatorname{codim}\left(\operatorname{Im}\left(U_{f, p}(T, 0)-I\right)\right)-1 .
$$

In both cases, we conclude that $D_{t, u} \Phi(T, p(0), f)$ is a Fredholm operator of index 1. We notice that, if $D_{t, u} \Phi(T, p(0), f)$ is a surjective map from $\mathbb{R} \times H^{s}\left(S^{1}\right)$ into itself, then the dimension of $\left.\operatorname{Ker} D_{t, u} \Phi(T, p(0), f)\right)$ is equal to 1 . We also remark that if $T$ is a simple period of $p(t)$, then $D_{t, u} \Phi(T, p(0), f)$ is a surjective map. In particular, for any $(T, p(0), f) \in \Phi^{-1}(0)$ such that the least period of $S_{f}(t) p(0)$ is less than (or equal to) $(3 / 2)^{k-1} \varepsilon$, we know that $D_{t, u} \Phi(T, p(0), f)$ is a surjective map, since $f$ belongs to $\mathcal{P}(k-1)$.

We next show that hypothesis ii) of Theorem A.1 is also satisfied. By the above considerations, we are reduced to proving that $D_{t, u, f} \Phi(T, p(0), f)$ is a surjective 
map, only in the case where $T \in\left[(3 / 2)^{k-1} \varepsilon,\left((3 / 2)^{k}+\delta^{*}\right) \varepsilon\right]$ is the least period of $p(t)=S_{f}(t) p(0)$. An easy computation shows that

$$
D_{t, u, f} \Phi(T, p(0), f)(\tau, v, g)=p_{t}(T) \tau+\left(U_{f, p}(T, 0)-I\right) v+\Sigma_{f, p}(T) g,
$$

where $\Sigma_{f, p}(t) g=w(t)$ is the solution of the following affine equation:

$$
\begin{aligned}
& w_{t}(x, t)=w_{x x}(x, t)+D_{u} f\left(x, p, p_{x}\right) w+D_{u_{x}} f\left(x, p, p_{x}\right) w_{x}+g\left(x, p, p_{x}\right), \\
& w(x, 0)=0 .
\end{aligned}
$$

We remark that

$$
w(t)=\int_{0}^{t} U_{f, p}(t, s) g\left(\cdot, p(s), p_{x}(s)\right) d s .
$$

The map $D_{t, u, f} \Phi(T, p(0), f)$ is surjective if and only if, for any $h$ in $H^{s}\left(S^{1}\right)$, there exists $(\tau, v, g) \in \mathbb{R} \times H^{s}\left(S^{1}\right) \times R \mathfrak{G}$ such that

$$
p_{t}(T) \tau+\left(U_{f, p}(T, 0)-I\right) v+\Sigma_{f, p}(T) g=h .
$$

Due to the Fredholm alternative, $h-\Sigma_{f, p}(T) g$ belongs to the image of $U_{f, p}(T, 0)-I$ if and only if

$$
\int_{S^{1}}\left(h-\Sigma_{f, p}(T) g\right) \varphi^{*}(x) d x=0
$$

for any solution $\varphi^{*}$ of the adjoint equation

$$
\left(U_{f, p}(T, 0)\right)^{*} \varphi^{*}=\varphi^{*} .
$$

Let $\varphi_{i}^{*}$, be a (at most two-dimensional) basis of $\operatorname{Ker}\left(U_{f, p}(T, 0)-I d\right)^{*}$. We must find $g$ such that $\int_{S^{1}}\left(\Sigma_{f, p}(T) g\right) \varphi_{i}^{*}(x) d x=\int_{S^{1}} h(x) \varphi_{i}^{*}(x) d x$, for any $i$. The surjectivity of the map $g \mapsto\left(\int_{S^{1}}\left(\Sigma_{f, p}(T) g\right) \varphi_{i}^{*}(x) d x\right)_{i}$ is equivalent to the non-existence of $\left(c_{i}\right)_{i}$ such that $\sum_{i} c_{i} \int_{S^{1}}\left(\Sigma_{f, p}(T) g\right) \varphi_{i}^{*}(x) d x=0$. Thus, we are reduced to proving that there is no solution $\varphi^{*} \neq 0$ of the adjoint equation $\left(U_{f, p}(T, 0)\right)^{*} \varphi^{*}=\varphi^{*}$ such that $\int_{S^{1}}\left(\Sigma_{f, p}(T) g\right) \varphi^{*}(x) d x=0$, for every $g \in R \mathfrak{G}$. In other words, we are reduced to prove that, for any solution $\varphi^{*} \neq 0$ of the adjoint equation $\left(U_{f, p}(T, 0)\right)^{*} \varphi^{*}=\varphi^{*}$, there exists $g \in R \mathfrak{G}$ such that

$$
\int_{S^{1}}\left(\Sigma_{f, p}(T) g\right) \varphi^{*}(x) d x \neq 0
$$

Using the property (4.7), we see that the condition (4.10) is equivalent to the fact that, for any $\varphi^{*}$, there exists $g \in R \mathfrak{G}$ such that

$$
\begin{aligned}
& \int_{S^{1}} \int_{0}^{T} U_{f, p}(T, s)(g\left.\left(x, p(x, s), p_{x}(x, s)\right)\right) \varphi^{*}(x) d s d x \\
&=\int_{S^{1}} \int_{0}^{T} g\left(x, p(x, s), p_{x}(x, s)\right)\left[\left(U_{f, p}(T, s)\right)^{*} \varphi^{*}\right](x) d s d x \neq 0 .
\end{aligned}
$$

Since $\varphi^{*} \neq 0$, there exist $x_{0} \in S^{1}$ and $t_{0} \in[0, T)$ such that $\left[\left(U_{f, p}\left(T, t_{0}\right)\right)^{*} \varphi^{*}\right]\left(x_{0}\right) \neq$ 0 . It is easy to construct a regular bump function $g\left(x, u, u_{x}\right)$ which vanishes outside a small neighborhood of $\left(x_{0}, p\left(x_{0}, t_{0}\right), p_{x}\left(x_{0}, t_{0}\right)\right)$ and is positive in this neighborhood. Due to the injectivity property of Lemma 4.2 for $x_{0}$ fixed, there exists no other time $t_{1} \in[0, T)$ such that $p\left(x_{0}, t_{1}\right)=p\left(x_{0}, t_{0}\right)$ and $p_{x}\left(x_{0}, t_{1}\right)=p_{x}\left(x_{0}, t_{0}\right)$. Therefore, the function $(x, s) \longmapsto g\left(x, p(x, s), p_{x}(x, s)\right)$ is a regular bump function 
concentrated around $\left(x_{0}, t_{0}\right)$. For such a choice of $g$, the condition (4.11) is thus satisfied.

Since all the hypotheses of Theorem A.1 hold, there exists a generic subset $V_{1}$ of $V$ such that, for any $f \in V_{1}$, the map $D_{t, u} \Phi(T, p(0), f)$ is a surjective map. If $p_{t}(T)$ belongs to $\operatorname{Im}\left(U_{f, p}(T, 0)-I\right)$, then $U_{f, p}(T, 0)-I$ is surjective, which is not possible since it is a Fredholm operator of index 0 and its kernel contains $p_{t}(0)$. Thus, $p_{t}(T)$ does not belong to $\operatorname{Im}\left(U_{f, p}(T, 0)-I\right)$ and 1 is an eigenvalue of algebraic multiplicity 1. Then, $\operatorname{dim}\left(\operatorname{Ker}\left(U_{f, p}(T, 0)-I\right)\right)=\operatorname{codim}\left(\operatorname{Im}\left(U_{f, p}(T, 0)-I\right)\right)=1$, and thus 1 is an eigenvalue of geometric multiplicity 1 . The proposition is then proved.

\section{GENERIC HYPERBOLICITY FOR NON-LINEARITIES INDEPENDENT OF $x$}

The purpose of this section is the proof of Theorem 1.3. We denote by $\mathfrak{G}^{i}$ the subspace of $\mathfrak{G}$ consisting of functions independent of $x$. We use the terminology of Proposition [1.1. Notice that if $u$ is a frozen wave, then every spatial translation $u\left(\cdot-x_{0}\right)$ of $u$ is also a frozen wave. This means that a frozen wave always belongs to a circle of equilibria and is never a hyperbolic equilibrium of (1.2). However, it is more natural to consider the frozen waves as particular cases of rotating waves with speed $c=0$. For the remaining part of this section, we group the waves with speed $c \in \mathbb{R}$ into a single category and simply call them "waves" if there is no need to distinguish between rotating and frozen waves. To simplify the notation, we say that a frozen wave $u$ is hyperbolic if the corresponding circle of equilibria is normally hyperbolic; that is, if 0 is a simple eigenvalue of the linearized operator with the eigenfunction $u_{x}$ and is the only eigenvalue with real part equal to 0 .

If $u(x, t)=v(x-c t)$ is a wave solution of (1.2) of speed $c \in \mathbb{R}$, then $v$ is an equilibrium of the equation

$$
w_{t}=w_{x x}+f\left(w, w_{x}\right)+c w_{x} .
$$

For this reason, the first step of the proof of Theorem 1.3 consists in eliminating the time dependence in the problem. Let $L: H^{2}\left(S^{1}\right) \longrightarrow L^{2}\left(S^{1}\right)$ be the linearized operator defined by

$$
L \varphi=\varphi_{x x}+f_{u}^{\prime}\left(v, v_{x}\right) \varphi+f_{u_{x}}^{\prime}\left(v, v_{x}\right) \varphi_{x}+c \varphi_{x} .
$$

Lemma 5.1. A wave $u(x, t)=v(x-c t)$ of (1.2) is hyperbolic if and only if 0 is a simple eigenvalue of $L$. Moreover, if $v$ is spatially $\frac{1}{n}$-periodic, i.e. $t \mapsto u(t)$ is $\frac{1}{n c}$ periodic, then any solution $\psi$ of $L \psi=0$ or of $L^{*} \psi=0$ is also spatially periodic of period $\frac{1}{n}$.

Proof. If $u$ is a frozen wave, then the first assertion of Lemma 5.1 is a direct consequence of Proposition 2.3 .

Let $u(x, t)=v(x-c t)$ be a rotating wave of period $T=1 / c$. We consider the operator $U(T, 0): H^{s}\left(S^{1}\right) \longrightarrow H^{s}\left(S^{1}\right)$ defined by $U(T, 0) \varphi_{0}=\varphi(T)$, where $\varphi(t)$ is the solution of

$$
\left\{\begin{array}{l}
\varphi_{t}=\varphi_{x x}+f_{u}^{\prime}\left(u, u_{x}\right) \varphi+f_{u_{x}}^{\prime}\left(u, u_{x}\right) \varphi_{x} \\
\varphi(x, 0)=\varphi_{0}(x)
\end{array}\right.
$$

We set $\varphi(x, t)=\psi(x-c t, t)$ so that $\psi$ satisfies $\psi_{t}=L \psi$. Let $V(T, 0)=e^{T L}$. Since $c T=1$, there is a perfect correspondence between the spectrum of $U(T, 0)$ and the spectrum of $V(T, 0)$. In particular, Proposition 2.5 shows that the rotating wave $u$ is hyperbolic if and only if 1 is a simple eigenvalue of $V(T, 0)$. Notice that $v_{x} \neq 0$ belongs to Ker $L$. Thus, by Proposition 2.3 zero is the only eigenvalue of zero real 
part for $L$. The spectral theorem recalled in (2.4) implies that $u$ is hyperbolic if and only if 0 is a simple eigenvalue of $L$.

Assume now that $v$ is $\frac{1}{n}$-periodic. Let $\tilde{L}$ be the restriction of $L$ to $H_{1 / n-p e r}^{s}\left(S^{1}\right)=$ $H^{s}(\mathbb{R} /(1 / n) \mathbb{Z})$ and let $\tilde{v}_{x}$ be the restriction of $v_{x}$ to $\mathbb{R} /(1 / n) \mathbb{Z}$. We have $\tilde{L} \tilde{v}_{x}=0$, which implies that 0 is also an eigenvalue of $\tilde{L}^{*}$. By Proposition 2.3 , the only eigenvalue of $\tilde{L}$ or $\tilde{L}^{*}$ with zero real part is zero. Using the remark following Proposition 2.5. we prove that every solution of $L \psi=0$ (resp. $\left.L^{*} \psi=0\right)$ is necessarily $\frac{1}{n}$-periodic and a solution of $\tilde{L} \tilde{\psi}=0$ (resp. $\left.\tilde{L}^{*} \tilde{\psi}=0\right)$.

We have to consider the case of homogeneous equilibria separately.

Lemma 5.2. There exists a generic dense subset $\mathcal{O}^{\text {hom }}$ of $\mathfrak{G}^{i}$ such that every homogeneous equilibrium point of (1.2) is hyperbolic.

Proof. The lemma is a direct consequence of the following computation. If $u \in \mathbb{R}$ is a homogeneous equilibrium, then there exist a function $\varphi \in H^{2}\left(S^{1}\right)$ and $\mu \in \mathbb{R}$ such that $\varphi_{x x}+f_{u}^{\prime}(u, 0) \varphi+f_{u_{x}}^{\prime}(u, 0) \varphi_{x}=i \mu \varphi$ if and only if there exists $k \in \mathbb{Z}$ such that $2 k f_{u_{x}}^{\prime}(u, 0) \pi=\mu$ and $f_{u}^{\prime}(u, 0)=4 k^{2} \pi^{2}$. Thus $\mathcal{O}^{\text {hom }}=\left\{f \in \mathfrak{G}^{i} \mid f(u, 0)=0 \Rightarrow\right.$ $\left.f_{u}^{\prime}(u, 0) \notin 4 \pi^{2} \mathbb{N}\right\}$ is a suitable choice.

The main step of the proof of Theorem 1.3 consists in applying Sard-Smale Theorem to deal with the waves.

Lemma 5.3. There exists a generic subset $\mathcal{O}^{\text {wav }}$ of $\mathfrak{G}^{i}$ such that every wave of (1.2) is hyperbolic.

Proof. The proof is very similar to the one of Proposition 4.3. The main tool is Theorem A.1 of the appendix. We recall that $\mathfrak{G}^{i}$ is not a metrizable space. To overcome this difficulty, we write $\mathcal{O}^{\text {wav }}=\cap_{n \in \mathbb{N}} \mathcal{O}_{n}^{\text {wav }}$ where $\mathcal{O}_{n}^{\text {wav }}$ is the set of functions of $\mathfrak{G}^{i}$ such that every wave $v$ of (1.2) with $\|v\|_{C^{1}}<n$ is hyperbolic. We next prove that, for each $n \in \mathbb{N}, \mathcal{O}_{n}^{\text {wav }}$ is a generic subset of $\mathfrak{G}^{i}$, which implies that $\mathcal{O}^{\text {wav }}$ is a generic subset of $\mathfrak{G}^{i}$, since it is a countable intersection of generic sets.Let $n \in \mathbb{N}$. We again introduce the restriction operator $R: g \in \mathfrak{G}^{i} \mapsto R g \in$ $C^{2}([-(n+2), n+2] \times[-(n+2), n+2], \mathbb{R})$ defined by

$$
R g=g_{\mid[-(n+2), n+2] \times[-(n+2), n+2]} .
$$

We remind that $R \mathfrak{G}^{i}=C^{2}([-(n+2), n+2] \times[-(n+2), n+2], \mathbb{R})$ is a separable Banach space. As in the proof of Proposition 4.3 it is sufficient to work with $C^{2}([-(n+2), n+2] \times[-(n+2), n+2], \mathbb{R})$ since the values of $f$ outside $[-n, n] \times[-n, n]$ do not matter for the genericity of the set $\mathcal{O}_{n}^{\text {wav }}$.

We apply Theorem A.1 of the appendix with the following spaces and functional. We set $U=\left\{v \in H^{2}\left(S^{1}\right) \mid v_{x} \not \equiv 0,\|v\|_{C^{1}}<n\right\} \times \mathbb{R}, V=R \mathfrak{G}^{i}$ and $Z=L^{2}\left(S^{1}\right)$. We set $z=0$ and

$$
\Phi:\left(\begin{array}{ccc}
U \times V & \longrightarrow & Z \\
(v, c, f) & \longmapsto & v_{x x}+f\left(v, v_{x}\right)+c v_{x}
\end{array}\right) .
$$

First notice that hypothesis iii) of Theorem A.1 is satisfied and that a point $(v, c, f)$ belongs to $\Phi^{-1}(0)$ if and only if $u(x, t)=v(x-c t)$ is a wave of speed $c$ for (1.2). 
We set

$$
L \varphi=\varphi_{x x}+f_{u}^{\prime}\left(v, v_{x}\right) \varphi+f_{u_{x}}^{\prime}\left(v, v_{x}\right) \varphi_{x}+c \varphi_{x} .
$$

We recall that, as proved in Lemma 2.2. $L$ is a Fredholm operator of index 0 .

Hypothesis i) of Theorem A.1 holds. Indeed, $D_{v, c} \Phi(v, c, f) \cdot(\varphi, d)=L \varphi+d v_{x}$. As in the proof of Proposition 4.3. there are two cases. Either $v_{x}$ belongs to $\operatorname{Im}(L)$ and thus $\operatorname{dim}\left(\operatorname{Ker}\left(D_{v, c} \Phi\right)\right)=\operatorname{dim}(\operatorname{Ker}(L))+1, \operatorname{codim}\left(\operatorname{Im}\left(D_{v, c} \Phi\right)\right)=\operatorname{codim}(\operatorname{Im}(L))$ or $v_{x}$ is not in $\operatorname{Im}(L)$ and then $\operatorname{dim}\left(\operatorname{Ker}\left(D_{v, c} \Phi\right)\right)=\operatorname{dim}(\operatorname{Ker}(L))$ and $\operatorname{codim}\left(\operatorname{Im}\left(D_{v, c} \Phi\right)\right)=$ $\operatorname{codim}(\operatorname{Im}(L))-1$. In both cases, since $L$ is Fredholm of index $0, D_{v, c} \Phi$ is a Fredholm operator of index 1.

Hypothesis ii) of Theorem A.1 is also satisfied. Indeed, let $h \in L^{2}\left(S^{1}\right)$. We have to find $v$ and $g$ such that $D \Phi(v, c, f) \cdot(\varphi, 0, g)=L \varphi+g\left(v, v_{x}\right)=h$. Due to the Fredholm alternative, $h-g\left(v, v_{x}\right)$ belongs to $\operatorname{Im}(L)$ if and only if $\int_{S^{1}}\left(h-g\left(v, v_{x}\right)\right) \psi=$ 0 for all $\psi$ solutions of $L^{*} \psi=0$. Let $\left(\psi_{i}\right)$ be a (at most two-dimensional) basis of $\operatorname{Ker}\left(L^{*}\right)$; we must find $g$ such that $\int g\left(v, v_{x}\right) \psi_{i}=\int h \psi_{i}$ for all $i$. The surjectivity of the map $g \mapsto\left(\int g\left(v, v_{x}\right) \psi_{i}\right)_{i}$ is equivalent to the non-existence of $\left(c_{i}\right) \neq 0$ such that $\sum c_{i} \int g\left(v, v_{x}\right) \psi_{i}=0$ for all $g$. Thus, setting $\psi=\sum c_{i} \psi_{i}$, we are reduced to proving that there is no $\psi \neq 0$ such that $L^{*} \psi=0$ and $\int_{S^{1}} g\left(v, v_{x}\right) \psi=0$ for all $g \in R \mathfrak{G}^{i}$. Let $\psi$ satisfy $L^{*} \psi=0$ and $\int_{S^{1}} g\left(v, v_{x}\right) \psi=0$ for all $g \in R \mathfrak{G}^{i}$. Let $m \neq 0$ be the integer such that $1 / m$ is the least period of the function $v$. By Lemma [5.1, $\psi$ is also $1 / m$-periodic and $\int_{S^{1}} g\left(v, v_{x}\right) \psi=0$ is equivalent to $\int_{0}^{1 / m} g\left(v, v_{x}\right) \psi=0$. Since $v$ is a periodic solution of least period $1 / m$ of a second order ordinary differential equation, the map $x \in[0,1 / m) \mapsto\left(v(x), v_{x}(x)\right) \in \mathbb{R}^{2}$ is injective and, hence, $\int_{0}^{1 / m} g\left(v, v_{x}\right) \psi=0$ for all $g$ implies that $\psi \equiv 0$. This shows the surjectivity of $D \Phi(v, c, f)$.

All the assumptions of Theorem A.1 being satisfied, there exists a generic subset $R \mathcal{O}^{\text {wav }}$ of $R \mathfrak{G}^{i}$ such that for any $f \in R \mathcal{O}_{n}^{\text {wav }}$ and for any wave $u(x, t)=v(x-c t)$ of (1.2) in $U$, the map $D_{v, c} \Phi:(\varphi, d) \mapsto L \varphi+d v_{x}$ is surjective. If $v_{x}$ belongs to $\operatorname{Im}(L)$, this surjectivity implies that $L$ is surjective, which is not possible since $L$ is a Fredholm operator of index 0 and since $\operatorname{Ker}(L)$ is not $\{0\}$ because it contains $v_{x} \neq 0$. Thus, $v_{x}$ does not belongs to $\operatorname{Im}(L)$, which means that 0 is an algebraically simple eigenvalue for $L$. Moreover, $\operatorname{dim}(\operatorname{Ker} L)=\operatorname{codim}(\operatorname{Im}(L))=1$, which means that 0 is a geometrically simple eigenvalue of $L$. The conclusion is then given by Lemma 5.1 .

Proof of Theorem 1.3. Applying Lemmas 5.2 and 5.3, we obtain a generic subset of $\mathfrak{G}^{i}$ such that all homogeneous equilibria and all waves of (1.2) are hyperbolic. To obtain Theorem 1.3 it only remains to remove the frozen waves. Indeed, we recall that a frozen wave is never hyperbolic as a non-homogeneous equilibrium point of (1.2) (and not as a wave, as it was considered in the above lemmas). As already noticed in [13], if we replace $f\left(u, u_{x}\right)$ by $f\left(u, u_{x}\right)+\varepsilon u_{x}$, we can "unfreeze" every frozen wave. To be sure that we are not "freezing" some rotating wave, we remark that there is at most a countable number of hyperbolic waves. Indeed, by arguments similar to the ones used in the proof of property (d) of Proposition 4.1 one can show that there is a finite number of periodic orbits $p$ of period less than $n$ and satisfying $\sup _{t}\|p(t)\|_{\mathcal{C}^{1}} \leq n$. Thus, we can choose $\varepsilon>0$ as small as is wanted to unfreeze the frozen waves without freezing any rotating wave.

We emphasize that, for a general system, without the constraint on the period, the number of hyperbolic periodic orbits could be infinite, even in bounded sets, 
since hyperbolic periodic orbits might pile up on a homoclinic orbit of a hyperbolic equilibrium point. In the case of Equation (1.2), one should be able to remove the constraint on the period, since there do not exist homoclinic orbits (see [13]).

\section{A. Appendix: Sard-Smale Theorem}

Let $M$ and $M^{\prime}$ be two differentiable Banach manifolds and let $f: M \longrightarrow M^{\prime}$ be a differentiable map. We say that $y \in M^{\prime}$ is a regular value of $f$ if, for any $x \in M$ such that $f(x)=y$, the differential $D f(x): T_{x} M \longrightarrow T_{y} M^{\prime}$ is surjective. The points of $M^{\prime}$ which are not regular are said to be critical. The classical theorem of Sard says that if $U$ is an open set of $\mathbb{R}^{p}$ and if $f: U \longrightarrow \mathbb{R}^{q}$ is of class $\mathcal{C}^{s}$ with $s>$ $\max (p-q, 0)$, then the set of critical values of $f$ in $\mathbb{R}^{q}$ is of Lebesgue measure zero. Using Fredholm operators and a Lyapunov-Schmidt method, Smale has generalized the Sard Theorem to infinite-dimensional spaces (for Fredholm operators, we refer to [5]). The Sard-Smale Theorem stated below is an application of the Smale Theorem.

Theorem A.1. Let $X, Y, Z$ be three smooth Banach manifolds. Let $U \subset X, V \subset Y$ be two open sets, $\Phi: U \times V \longrightarrow Z$ be a map of class $\mathcal{C}^{k}(k \geq 1)$ and $z$ be a point of $Z$.

We assume that:

i) $\forall(x, y) \in \Phi^{-1}(z), D_{x} \Phi(x, y)$ is a Fredholm operator of index strictly less than $k$,

ii) $\forall(x, y) \in \Phi^{-1}(z), D \Phi(x, y)$ is surjective,

iii) $X$ and $Y$ are separable.

Then $\Theta=\{y \in V \mid z$ is a regular value of $\Phi(., y)\}$ is a generic subset of $V$.

The proof of Theorem A.1 can be found in 28] or [27] (for stronger versions, see also [17]). We also refer to [19], where one can find a short review on PDE generic results, as well as adaptations of the transversality theorems to the notion of prevalence, which is a notion of "almost always" different from the genericity.

\section{ACKNOWLEDGEMENTS}

The authors wish to thank P. Brunovský, R. Czaja and C. Rocha for fruitful discussions with them.

\section{REFERENCES}

1. S. Angenent, The Morse-Smale property for a semi-linear parabolic equation, J. Differential Equations $\mathrm{n}^{\mathrm{O}} 62$ (1986), pp. 427-442. MR837763(87e:58115)

2. S. Angenent, The zero set of a solution of a parabolic equation, Journal für die Reine und Angewandte Mathematik no390 (1988), pp. 79-96. MR.953678 (89j:35015)

3. S. Angenent and B. Fiedler, The dynamics of rotating waves in scalar reaction diffusion equations, Transaction of the American Mathematical Society $\mathrm{n}^{\circ} 307$ (1988), pp. 545-568. MR 940217 (89h:35158)

4. A.V. Babin and M.I. Vishik, Attractors of evolution equations, Studies in Mathematics and its Applications no 25 (1992), North-Holland. MR.1156492 (93d:58090)

5. R.A. Bonic, Linear functional analysis, Gordon and Breach Science Publishers (1969). MR 0257686 (41:2336)

6. P. Brunovský and S-N. Chow, Generic properties of stationary state solutions of reactiondiffusion equations, Journal of Differential Equations $\mathrm{n}^{\circ} 53$ (1984), pp. 1-23. MR747403 (86a:35072) 
7. P. Brunovský and P. Poláčik, The Morse-Smale structure of a generic reaction-diffusion equation in higher space dimension, Journal of Differential Equations no ${ }^{\circ} 135$ (1997), pp. 129181. MR1434918 (98e:35091)

8. P. Brunovský and G. Raugel, Genericity of the Morse-Smale property for damped wave equations, Journal of Dynamics and Differential Equations, vol $15 \mathrm{n}^{\circ} 2$ (2003), pp. 571-658. MR2046732 (2005b:37184)

9. R. Czaja and C. Rocha, Transversality in scalar reaction-diffusion equations on a circle, Journal of Differential Equations n ${ }^{\circ} 245$ (2008), pp. 692-721.

10. B. Fiedler and J. Mallet-Paret, A Poincaré-Bendixson theorem for scalar reaction-diffusion equations, Arch. Rational Mech. Analysis no 107 (1989), pp. 325-345. MR1004714 (90j:35116)

11. B. Fiedler, C. Rocha, Heteroclinic orbits of semilinear parabolic equations, Journal of Differential Equations no 156 (1996), pp. 239-281. MR.1376067 (96k:58200)

12. B. Fiedler, C. Rocha, Orbit equivalence of global attractors of semilinear parabolic differential equations, Trans. Amer. Math. Soc. $\mathrm{n}^{\circ} 352$ (2000), pp. 257-284. MR1475682 (2000c:35129)

13. B. Fiedler, C. Rocha and, M. Wolfrum, Heteroclinic orbits between rotating waves of semilinear parabolic equations on the circle, Journal of Differential Equations n ${ }^{\circ} 201$ (2004), pp. 99-138. MR2057540 (2005f:37172)

14. M. Golubitsky and V. Guillemin, Stable mapping and their singularities, Graduate Texts in Mathematics $n^{o} 14$, Springer-Verlag, New York-Heidelberg (1973). MR0341518 (49:6269)

15. D. Henry, Geometric theory of semilinear parabolic equations, Lecture Notes In Math. $\mathrm{n}^{\circ} 840$ Springer-Verlag (1981). MR610244 (83j:35084)

16. D. Henry, Some infinite dimensional Morse-Smale systems defined by parabolic differential equations, J. Diff. Equat. n 59 (1985), pp. 165-205. MR804887 (86m:58080)

17. D. Henry, Perturbation of the Boundary for Boundary Value Problems of Partial Differential Operators, with editorial assistance from Jack Hale and Antonio Luiz Pereira. London Mathematical Society Lecture Note Series $\mathrm{n}^{\circ} 318$. Cambridge University Press, Cambridge UK (2005). MR2160744 (2006f:35003)

18. R. Joly, Generic transversality property for a class of wave equations with variable damping, Journal de Mathématiques Pures et Appliquées no84 (2005), pp. 1015-1066. MR2155898 (2007j:35138)

19. R. Joly, Adaptation of the generic PDE's results to the notion of prevalence, J. Dynamics and Differential Equations n ${ }^{\circ} 19$ (2007), pp. 967-983. MR2357534

20. R. Joly and G. Raugel, Generic Morse-Smale property for the parabolic equation on the circle, submitted.

21. J. Mallet-Paret, Generic periodic solutions of functional differential equations, J. Diff. Equations $\mathrm{n}^{\circ} 25,(1977)$, pp. 163-183. MR0442995 (56:1370)

22. H. Matano, Nonincrease of the lap-number of a solution for a one-dimensional semi-linear parabolic equation, J. Fac. Sci. Univ. Tokyo Sec. IA, no 29 (1982), pp. 221-227. MR672070 (84m:35060)

23. H. Matano and K.-I. Nakamura, The global attractor of semilinear parabolic equations on $S^{1}$, Discrete and Continuous Dyn. Syst. n³ (1997), pp. 1-24. MR1422536 (97g:35072)

24. J. Palis and W. de Melo: Geometric Theory of Dynamical Systems, Springer-Verlag, Berlin (1982). MR.669541 (84a:58004)

25. A. Pazy, Semigroups of Linear Operators and Applications to Partial Differential Equations, Appl. Math. Sci. nº44, Springer, New York (1983). MR710486 (85g:47061)

26. M. M. Peixoto, On an approximation theorem of Kupka and Smale, J. Diff. Equations $\mathrm{n}^{\circ} 3$ (1966), pp. 214-227. MR0209602 (35:499)

27. F. Quinn, Transversal approximation on Banach manifolds, Global Analysis (Proceedings of Symposia in Pure Mathematics $\mathrm{n}^{\circ} 15$, Berkeley, 1968) pp. 213-222, Amer. Math. Soc., Providence (1970). MR0264713 (41:9304)

28. J.C. Saut and R. Temam, Generic properties of nonlinear boundary value problems, Communications in PDE n⿳0 (1979), pp. 293-319. MR.522714 (80i:35080)

29. B. Sandstede and B. Fiedler, Dynamics of periodically forced parabolic equations on the circle, Ergodic Theory and Dynamical Systems no 12 (1992), pp. 559-571. MR1182662 (93h:35103)

30. J. Smoller and A. Wasserman, Generic properties of steady state solutions, J. Diff. Equat. $\mathrm{n}^{\circ} 52$ (1984), pp. 423-438. MR744306 (86h:35013)

31. C. Sturm, Sur une classe d'équations à différences partielles, J. Math. Pures Appl. $\mathrm{n}^{\circ} 1$ (1836), pp. 373-444. 
32. T. J. Zelenyak, Stabilization of solutions of boundary value problems for a second order parabolic equation with one space variable, Diff. Equations $\mathrm{n}^{\circ} 4$ (1968), pp. 17-22. MR 0223758 (36:6806)

Institut Fourier, UMR CNRS 5582, Université De Grenoble I, B.P. 74, 38402 SaintMartin-D'HÈres, France

E-mail address: Romain.Joly@ujf-grenoble.fr

CNRS, Laboratoire de Mathématiques d'Orsay, Université Paris-Sud, F-91405 Orsay Cedex, France

E-mail address: Genevieve.Raugel@math.u-psud.fr 